\title{
Optimal risk sharing for law invariant monetary utility functions
}

\author{
E. Jouini ${ }^{*} \quad$ W. Schachermayer ${ }^{\dagger} \quad$ N. Touzi ${ }^{\ddagger}$
}

April 19, 2007

\begin{abstract}
We consider the problem of optimal risk sharing of some given total risk between two economic agents characterized by law-invariant monetary utility functions or equivalently, law-invariant risk measures. We first prove existence of an optimal risk sharing allocation which is in addition increasing in terms of the total risk. We next provide an explicit characterization in the case where both agents' utility functions are comonotone. The general form of the optimal contracts turns out to be given by a sum of options (stop-loss contracts, in the language of insurance) on the total risk. In order to show the robustness of this type of contracts to more general utility functions, we introduce a new notion of strict risk aversion conditionally on lower tail events, which is typically satisfied by the semi-deviation and the entropic risk measures. Then, in the context of an AV@R-agent facing an agent with strict monotone preferences and exhibiting strict risk aversion conditional on lower tail events, we prove that optimal contracts again are European options on the total risk.
\end{abstract}

MSC 1991 subject classifications: Primary 91B06, 46A20; secondary 91B70.

Key words : Monetary utility functions, comonotonicity, Pareto optimal allocations.

\footnotetext{
${ }^{*}$ Université Paris Dauphine and CEREMADE, Place du Maréchal de Lattre de Tassigny, F-75775 Paris Cedex 16, France.

${ }^{\dagger}$ Vienna University of Technology, Wiedner Hauptstrasse 8-10/105, A-1040 Wien, Austria and Université Paris Dauphine, Place du Maréchal de Lattre de Tassigny, F-75775 Paris Cedex 16, France. Financial support from the Austrian Science Fund (FWF) under the grant P15889 and from Vienna Science and Technology Fund (WWTF) under Grant MA13 is gratefully acknowledged.

${ }^{\ddagger}$ CREST, Laboratoire de Finance et Assurance, 15 Bd Gabriel Péri, F-92245 Malakoff Cedex, France and Université Paris Dauphine, Place du Maréchal de Lattre de Tassigny, F-75775 Paris Cedex 16, France.
} 


\section{Introduction}

The problem of optimal sharing of risk between two agents has been considered by many authors, starting from Arrow [1], Borch [9], Bühlmann [7], Bühlmann and Jewell [8], see also Gerber [26]. The main motivation was the application to insurance problems. For an extensive list of references, we refer to the recent paper by Dana and Scarsini [15] and the second edition of the book by Föllmer and Schied [25]. The general setting of the problem is the following. The initial risk endowments of the agents are defined by the bounded random variables $X_{0}$ and $X_{1}$, so that the aggregate risk is given by $X:=X_{0}+X_{1}$. An allocation $\left(\xi_{0}, \xi_{1}\right)$, with $\xi_{0}+\xi_{1}=X$, is called an optimal risk sharing if it is Pareto optimal and satisfies the individual rationality constraint, i.e. none of the agents experiences a loss of utility by passing from $X_{i}$ to $\xi_{i}$.

The purpose of this paper is to obtain an explicit characterization of the optimal risk sharing in the context of monetary utility functions, see Definition 2.1 below. Up to the sign, monetary utility functions are identical to convex risk measures [24, 21], a popular notion in particular since the Basel II accord. Indeed, $U$ is a monetary utility function if and only if $\rho=-U$ is a convex risk measure in the sense of Föllmer and Schied [24], Frittelli and Rosazza-Gianin [21]. We shall rather use the language of monetary utility functions in order to embed our analysis in the setting of classical utility theory. Our framework is mainly motivated by Barrieu and El Karoui $[3,4,5,6]$ who showed that, when the agents' utilities are given by $U_{i}(\xi):=\gamma_{i}^{-1} U\left(\gamma_{i} \xi\right)$ for some $\gamma_{i}>0$ and some monetary utility function $U$, the optimal risk sharing rule is proportional to the aggregate risk. We also refer to Heath and $\mathrm{Ku}[27]$ for analyzing Pareto optimal risk between banks defined by coherent risk measures in a finite probability space.

In comparison to the general utility theory, the setting of monetary utility functions induces a remarkable simplification, as it induces a clear separation between the Pareto optimality and the individual rationality constraints which define an optimal risk sharing rule. Pareto optimal allocations are defined up to a constant, and their characterization reduces to the calculation of the sup-convolution of the utility functions, as observed in [3]. As a second independent step, the choice of the constant, or the premium, inside the interval of reservation prices of the agents then characterizes all optimal risk sharing allocations.

In this paper, we specialize further the class of monetary utility functions by assuming the law-invariance property. Under this condition, we prove that the set of optimal risk sharing allocations is not empty. We next derive an explicit characterization of optimal risk sharing allocations in two concrete settings :

(i) When the preferences of both agents are defined by comonotone law-invariant utility functions (a typical example being $U=-A V @ R_{\alpha}$ to be discussed below), a precise de- 
scription of the optimal risk sharing rule is provided. The key-ingredient is the quantile representation of Kusuoka [32] which is further extended in [22], [25] and [29]. We prove that Pareto optimal allocations are given by sums of European options ("stop-loss contracts" or simply "deductibles" in the insurance terminology) written on the aggregate risk $X$. We observe that this setting is intimately connected to the context of convex distorsions of probability as studied in Carlier and Dana [12].

(ii) As a second example, we fix some $\alpha \in(0,1)$, and we assume that the utility of Agent 0 is defined by $U_{0}=-A V @ R_{\alpha}$, where $A V @ R_{\alpha}$ is the so-called average value at risk or expected shortfall risk measure. This is the prime example of a comonotone monetary utility function. Agent 1 is defined by a law-invariant monetary utility function which is strictly monotone. We further assume that Agent 1 is strictly risk averse conditionally on lower tail events. This notion is defined in Section 3. Loosely speaking, it states that the agent has a strict preference for averaging lower tail events of risk. The class of monetary utility functions which satisfy these two conditions include the so-called entropic utility and the semi-deviation utility (see e.g. [23]). We remark that $U_{0}=-A V @ R_{\alpha}$ is neither strictly monotone, nor strictly risk averse conditionally on lower tail events. In the above setting, we show that Pareto optimal allocations are defined by classical options : Agent 0 offers a stop-loss contract defined by a threshold $\kappa$, and leaves Agent 1 with the aggregate risk capped at the level $\kappa$, i.e. $\left(\xi_{0}, \xi_{1}\right)=\left(-(X-\kappa)^{-}, \kappa \vee X\right)$. In particular this shows that Agent 0 takes the extremal risks, and that the AV@R measure of risk is not so prudent.

We believe that the above stated results provide an additional justification for the existence of options in financial markets, and stop-loss contracts, deductibles and layers in insurance markets.

We conclude this introduction by pointing out the importance of the concavity of the utility function. It was stressed in [2] that the V@R criterion for risk measuring leads to incoherent results because of the lack of sub-additivity. The present context of risk sharing provides another result in this direction. Indeed, let Agent 1 be defined by any monetary utility function. Assuming that Agent 0 is defined by the utility function $U_{0}=-V @ R_{\alpha}(X)$, it follows clearly that the level of the random variable on the event set $\left\{X<\mathrm{V} @ \mathrm{R}_{\alpha}(X)\right\}$ is not relevant for this agent. Then, an optimal risk sharing allocation

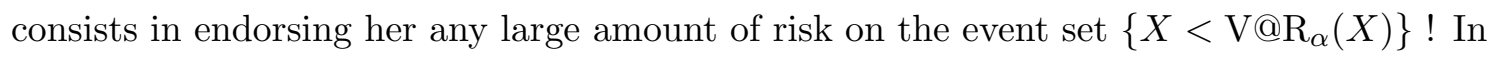
other words, the $V @ R_{\alpha}$-agent endorses an infinite amount of risk with positive probability. Hence the very question of optimal risk sharing leads to silly results if one of the agents uses the V@R criterion as a measure of risk.

The paper is organized as follows. Section 2 collects some notions on monetary utility functions. Our main results are stated in Section 3. Section 4 is devoted to the characterization of the optimal sharing allocations when both agents are defined by comonotone law-invariant monetary utility functions. In Section 5, we focus on the notion of strict 
risk aversion, conditional on lower tail events, together with its implications in terms of risk sharing. Finally, we report the existence of optimal risk sharing allocations under law-invariant monetary utility functions in Section 6.

\section{Preliminaries : utility functions and related notions}

Throughout this paper, we work on a standard probability space $(\Omega, \mathcal{F}, \mathbb{P})$, i.e. we suppose that $(\Omega, \mathcal{F}, \mathbb{P})$ has no atoms and that $\mathbb{L}^{2}(\Omega, \mathcal{F}, \mathbb{P})$ is separable. For every $p \in[0, \infty]$, we shall denote by $\mathbb{L}^{p}(\mathbb{P})$ the collection of all real-valued random variables with finite $\mathbb{L}^{p}$ norm under $\mathbb{P}$. Here, $\mathbb{L}^{0}:=\mathbb{L}^{0}(\mathbb{P})$ consists of all real-valued $\mathcal{F}$-measurable random variables, and $\mathbb{L}^{\infty}:=\mathbb{L}^{\infty}(\mathbb{P})$ is the subset of all essentially bounded elements in $\mathbb{L}^{0}$.

For every random variable $\xi$ with values in $\mathbb{R}$, we denote its cumulative distribution function by $F_{\xi}(x):=\mathbb{P}[\xi \leq x]$, and its generalized inverse (called quantile function) by $q_{\xi}(\alpha)=\inf \left\{x: F_{\xi}(x) \geq \alpha\right\}$ for $\alpha \in[0,1]$.

Given two random variables $\xi$ and $\zeta$, we shall write $\xi={ }_{d} \zeta$ to indicate equality in distribution.

For a bounded finitely additive measure $\mu \in\left(\mathbb{L}^{\infty}\right)^{*}$, we denote by $\|\mu\|_{\text {ba }}$ its total mass. Given a sub- $\sigma$-algebra $\mathcal{G}$ of $\mathcal{F}$, we define the conditional expectation $\left(\mathbb{L}^{\infty}\right)^{*} \ni \mu \longmapsto$ $\mathbb{E}[\mu \mid \mathcal{G}] \in\left(\mathbb{L}^{\infty}\right)^{*}$ as the transpose of the $\mathcal{G}$-conditional expectation operator on $\mathbb{L}^{\infty}$, i.e. $\langle\mathbb{E}[\mu \mid \mathcal{G}], \xi\rangle=\langle\mu, \mathbb{E}[\xi \mid \mathcal{G}]\rangle$ for all $\mu \in\left(\mathbb{L}^{\infty}\right)^{*}$ and $\xi \in \mathbb{L}^{\infty}$. If the singular part of $\mu$ is zero, i.e. $\mu$ is absolutely continuous with respect to $\mathbb{P}$ with density $d \mu / d \mathbb{P}=Z$, then it is immediately checked that this definition coincides with the classical notion of condition expectation in the sense that $\mathbb{E}[\mu \mid \mathcal{G}]=\mathbb{E}[Z \mid \mathcal{G}] \cdot \mathbb{P}$.

Let $E$ be a Banach space with dual $E^{*}$. Given a function $f: E \longrightarrow \mathbb{R}$, its sub-gradient and super-gradients are respectively denoted by

$$
\begin{aligned}
& \partial^{-} f(x):=\left\{x^{*} \in E^{*}: f(y) \geq f(x)+\left\langle x^{*}, y-x\right\rangle \text { for every } y \in E\right\}, \\
& \partial^{+} f(x):=\left\{x^{*} \in E^{*}: f(y) \leq f(x)+\left\langle x^{*}, y-x\right\rangle \text { for every } y \in E\right\} .
\end{aligned}
$$

When $f$ is convex (resp. concave), we shall simply denote $\partial f:=\partial^{-} f\left(\right.$ resp. $\left.\partial f:=\partial^{+} f\right)$.

\subsection{Monetary utility functions}

We say that a function $f: \mathbb{L}^{\infty} \longrightarrow[-\infty, \infty]$ is proper if $\operatorname{dom}(U):=\left\{\xi \in \mathbb{L}^{\infty}: U(\xi) \in \mathbb{R}\right\}$ is non-empty.

Definition 2.1. A proper function $U: \mathbb{L}^{\infty} \longrightarrow \mathbb{R}$ is called a monetary utility function if it is concave, monotone with respect to the order of $\mathbb{L}^{\infty}$, satisfies the normalization condition 
$U(0)=0$ and has the cash-invariance property

$$
U(\xi+c)=U(\xi)+c \quad \text { for every } \xi \in \mathbb{L}^{\infty} \text { and } c \in \mathbb{R} .
$$

Observe that the cash invariance and the monotonicity of $U$ imply that $U$ is finite and Lipschitz-continuous on $\mathbb{L}^{\infty}$. In particular, the normalization $U(0)=0$ does not restrict the generality as it may be obtained by adding a constant to $U$.

Monetary utility functions can be identified with convex risk measures by the formula $\rho(\xi)=-U(\xi)$. In particular, positively homogeneous monetary utility functions can be identified with coherent risk measures, introduced in Artzner, Delbaen, Eber and Heath [2], while the general notion of monetary utility functions corresponds to convex risk measures as introduced independently by Föllmer and Schied [24], and Frittelli and Rossaza-Gianin [21]. We shall frequently use the conjugate function

$$
V(\mu):=\sup _{\xi \in \mathbb{L}^{\infty}}(U(\xi)-\langle\mu, \xi\rangle) \in \mathbb{R} \cup\{\infty\}, \quad \mu \in\left(\mathbb{L}^{\infty}\right)^{*}
$$

which is convex and lower semi-continuous with respect to $\sigma\left(\left(\mathbb{L}^{\infty}\right)^{*}, \mathbb{L}^{\infty}\right)$. From classical convex duality theory, see e.g. [10], and the $\mathbb{L}^{\infty}$-continuity of $U$, it follows that $U$ and $V$ are $\left\langle\mathbb{L}^{\infty},\left(\mathbb{L}^{\infty}\right)^{*}\right\rangle$-conjugate, i.e.

$$
U(\xi)=\inf _{\mu \in\left(\mathbb{L}^{\infty}\right)^{*}}(V(\mu)+\langle\mu, \xi\rangle) \quad \text { for every } \xi \in \mathbb{L}^{\infty} .
$$

We shall denote by $V^{1}$ the restriction of $V$ to $\mathbb{L}^{1}(\mathbb{P})$, and remark that its domain is included in the set of densities of $\mathbb{P}$-absolutely continuous probability measures, i.e.

$$
\operatorname{dom}\left(V^{1}\right):=\left\{Z \in \mathbb{L}^{1}(\mathbb{P}): V(Z)<\infty\right\} \quad \subset \mathcal{Z}:=\left\{Z \in \mathbb{L}_{+}^{1}(\mathbb{P}): \mathbb{E}[Z]=1\right\}
$$

Theorem 2.1 ([19, 24]). For a monetary utility function $U$, the following statements are equivalent :

(i) $U$ and $V^{1}$ are $\left\langle\mathbb{L}^{\infty}, \mathbb{L}^{1}(\mathbb{P})\right\rangle$-conjugate, i.e. $U(\xi)=\inf _{Z \in \mathbb{L}^{1}(\mathbb{P})}\left(V^{1}(Z)+\mathbb{E}[Z \xi]\right)$ for $\xi \in \mathbb{L}^{\infty}$,

(ii) $U$ is $\sigma\left(\mathbb{L}^{\infty}, \mathbb{L}^{1}(\mathbb{P})\right)$ - upper semicontinuous,

(iii) $U$ has the Fatou property, i.e. for every $\mathbb{L}^{\infty}$-bounded sequence $\left(\xi_{n}\right)_{n \geq 1}$ converging in probability to some $\xi \in \mathbb{L}^{\infty}$, we have $U(\xi) \geq \lim \sup _{n} U\left(\xi_{n}\right)$.

Remark 2.1. Assuming the Fatou property, suppose that a monetary utility function $U$ is positively homogeneous, i.e. $U(\xi)=-\rho(\xi)$ for some coherent risk measure $\rho$. Then, the conjugate function $V$ is zero on its domain, and $U(X)=\inf _{Z \in \operatorname{dom}\left(V^{1}\right)} \mathbb{E}[Z X]$.

Given two monetary utility functions $U_{0}$ and $U_{1}$, we denote by

$$
U_{0} \square U_{1}(\xi):=\sup _{\xi_{0} \in \mathbb{L}^{\infty}} U_{0}\left(\xi_{0}\right)+U_{1}\left(\xi-\xi_{0}\right), \quad \xi \in \mathbb{L}^{\infty},
$$


the sup-convolution of the concave functions $U_{0}$ and $U_{1}$ (compare $[18,6]$ ). It is well known that $U_{0} \square U_{1}$ is a concave function on $\mathbb{L}^{\infty}$, see e.g. [36, 18]. Since $U_{0}$ and $U_{1}$ are finite on $\mathbb{L}^{\infty}$, it follows that $U_{0} \square U_{1}>-\infty$. Then, it follows from the concavity of $U_{0} \square U_{1}$ that

$$
\text { either } \operatorname{dom}\left(U_{0} \square U_{1}\right)=\mathbb{L}^{\infty}, \quad \text { or } \quad U_{0} \square U_{1} \equiv \infty \text {. }
$$

For later use, we provide the following well-known properties and their proof for completeness, see e.g. [6].

Lemma 2.1. Let $U_{0}$ and $U_{1}$ be two concave functions from $\mathbb{L}^{\infty}$ to $\mathbb{R}$ with associated convex conjugate functions $V_{0}$ and $V_{1}$ defined on $\left(\mathbb{L}^{\infty}\right)^{*}$, and assume that $U_{0} \square U_{1}$ is $\mathbb{L}^{\infty}$-continuous. Then:

(i) $\operatorname{dom}\left(U_{0} \square U_{1}\right)=\mathbb{L}^{\infty}$ if and only if $\operatorname{dom}\left(V_{0}\right) \cap \operatorname{dom}\left(V_{1}\right) \neq \emptyset$. In this case, $U_{0} \square U_{1}$ is a monetary utility function.

(ii) if $U_{0} \square U_{1}$ is proper, then $U_{0} \square U_{1}$ and $V_{0}+V_{1}$ are $\sigma\left\langle\left(\mathbb{L}^{\infty}\right)^{*}, \mathbb{L}^{\infty}\right\rangle-$ conjugate.

Proof. Assertion (i) is an immediate consequence of (ii). To see that (ii) holds true, we compute, similarly as in [3], that for every $\mu \in\left(\mathbb{L}^{\infty}\right)^{*}$ :

$$
\begin{aligned}
\sup _{\xi \in \mathbb{L}^{\infty}}\left(U_{0} \square U_{1}(\xi)-\langle\mu, \xi\rangle\right) & =\sup _{\xi \in \mathbb{L}^{\infty}} \sup _{\xi_{0} \in \mathbb{L}^{\infty}}\left(U_{0}\left(\xi_{0}\right)+U_{1}\left(\xi-\xi_{0}\right)-\langle\mu, \xi\rangle\right) \\
& =\sup _{\xi_{0}, \xi_{1} \in \mathbb{L}^{\infty}}\left(U_{0}\left(\xi_{0}\right)-\left\langle\mu, \xi_{0}+\xi_{1}\right\rangle+U_{1}\left(\xi_{1}\right)\right)=\left(V_{0}+V_{1}\right)(\mu) .
\end{aligned}
$$

Hence $V_{0}+V_{1}$ is the $\left\langle\left(\mathbb{L}^{\infty}\right)^{*}, \mathbb{L}^{\infty}\right\rangle$-conjugate of the upper-semicontinuous function $U_{0} \square U_{1}$. If $U_{0} \square U_{1}$ is proper, then (ii) follows from classical convex duality theory.

Remark 2.2. Given two monetary utility functions satisfying the Fatou property, notice that there is no guarantee in general for $U_{0} \square U_{1}$ to satisfy the Fatou property, see the counter-example reported in Delbaen [20] which we analyze in more detail in Section 6.3 below. Hence, in general, $U_{0} \square U_{1}$ is not the conjugate of $V_{0}^{1}+V_{1}^{1}$, where $V_{i}^{1}$ is the restriction of $V_{i}$ to $\mathbb{L}^{1}(\mathbb{P})$, for $i=0,1$.

\subsection{Law-invariance}

A function $f: \mathbb{L}^{\infty} \longrightarrow \mathbb{R}$ is said to be law-invariant if $f(\xi)$ depends only on the law of $\xi$ for every $\xi \in \mathbb{L}^{\infty}$.

Let $U$ be a law-invariant monetary utility function and $\xi \in \mathbb{L}^{\infty}$. By Lemma 3.2 of [29], $\mathbb{E}[\xi]$ lies in the $\sigma\left(\left(\mathbb{L}^{\infty}\right)^{*}, \mathbb{L}^{\infty}\right)$-closed convex hull of the set $\left\{\zeta \in \mathbb{L}^{\infty}: \zeta=_{d} \xi\right\}$. Then, it follows from the concavity and the law-invariance of $U$ that $U(\mathbb{E}[\xi]) \geq U(\xi)$ for any $\xi \in \mathbb{L}^{\infty}$, and therefore $V(1) \leq 0$. Since $U(0)=0$, this implies that

$$
1 \in \operatorname{dom}(V) \text { and } V(1)=0 .
$$

In particular, it follows from Lemma 2.1 that $\operatorname{dom}\left(U_{0} \square U_{1}\right)=\mathbb{L}^{\infty}$ for any pair $\left(U_{0}, U_{1}\right)$ of law-invariant monetary utility functions. 
Remark 2.3. This is a complement to Remark 2.2. Given two law-invariant monetary utility functions $U_{0}$ and $U_{1}$, we know from Lemma 2.1 and (2.2) that $U_{0} \square U_{1}$ is a monetary utility function. Clearly, $U_{0} \square U_{1}$ is law-invariant, so it has the Fatou property by [29]. Consequently $U_{0} \square U_{1}$ and $V_{0}^{1}+V_{1}^{1}$ are $\left(\mathbb{L}^{\infty}, \mathbb{L}^{1}(\mathbb{P})\right)$-conjugate.

An interesting characterization of law-invariant monetary utility functions, obtained by Kusuoka [32] and further extended in [22] and [29] (Theorem 2.1 and equation (14)), expresses any law-invariant coherent risk measure in terms of the quantile function. An important role is played by the set

$$
\mathcal{D}_{\searrow}:=\{g \in \mathcal{D}: g \text { non-increasing, right-continuous and } g(1)=0\}
$$

where $\mathcal{D}:=\left\{g:(0,1] \longrightarrow \mathbb{R}_{+}, \int_{0}^{1} g=1\right\}$ is the set of density functions on $(0,1]$.

Theorem 2.2 ([32, 22, 29]). The following statements are equivalent :

(i) $U$ is a law-invariant monetary utility function,

(ii) there is a convex function $v: \mathcal{D}_{\searrow} \longrightarrow \mathbb{R}_{+} \cup\{\infty\}$, with $\inf _{\mathcal{D} \searrow} v=0$, such that

$$
U(\xi)=\inf _{f \in \mathcal{D} \searrow} \int q_{\xi}(t) f(t) d t+v(f) \quad \text { for every } \quad \xi \in \mathbb{L}^{\infty} .
$$

For the purpose of this paper, it is convenient to introduce the set of primitive functions

$$
\mathcal{C} \backslash:=\left\{\varphi:[0,1] \longrightarrow[0,1]: \varphi(t)=\int_{0}^{t} g(s) d s \text { for some } g \in \mathcal{D} \searrow\right\}
$$

and its pointwise closure

$$
\overline{\mathcal{C}}_{\searrow}=\{\varphi:[0,1] \longrightarrow[0,1] \text { non-decreasing, concave, } \varphi(0)=0 \text { and } \varphi(1)=1\} .
$$

Clearly, $\mathcal{C} \backslash$ is the subset of all functions in $\overline{\mathcal{C}} \backslash$ which are continuous at the point $t=0$. Then, by a direct rephrasing of Theorem 2.2, we obtain a characterization of any lawinvariant monetary utility function $U$ as

$$
U(\xi)=\inf _{\varphi \in \mathcal{C} \backslash} \int q_{\xi}(t) \varphi^{\prime}(t) d t+v(\varphi)=\inf _{\varphi \in \overline{\mathcal{C}} \searrow} \int q_{\xi}(t) \varphi^{\prime}(t) d t+v(\varphi)
$$

for every $\xi \in \mathbb{L}^{\infty}$, where $v: \overline{\mathcal{C}} \backslash \longrightarrow \mathbb{R}_{+} \cup\{\infty\}$ is a convex function with $\inf _{\overline{\mathcal{C}} \backslash} v=0$. In the second equality of $(2.3), \varphi^{\prime}=\varphi(0+) \delta_{0}+\varphi^{\prime} \mathbf{1}_{(0,1]}$ in the distributional sense, so that $\varphi^{\prime}$ assigns a positive mass to the point $t=0$ whenever $\varphi \in \overline{\mathcal{C}} \backslash$ is not continuous at this point (see Remark 2.4 below).

\subsection{Comonotonicity}

Definition 2.2. Let $(\xi, \zeta)$ be a pair of random variables defined on $(\Omega, \mathcal{F}, \mathbb{P})$, with values in $\mathbb{R}$. 
(i) $\xi$ and $\zeta$ are comonotone, and we denote $\xi \sim_{c} \zeta$, if $\left(\xi\left(\omega_{1}\right)-\xi\left(\omega_{2}\right)\right)\left(\zeta\left(\omega_{1}\right)-\zeta\left(\omega_{2}\right)\right) \geq 0$ $\mathbb{P}(d \omega) \otimes \mathbb{P}\left(d \omega^{\prime}\right)-$ a.s.

(ii) $\xi$ increases with $\zeta$, and we denote $\xi / \zeta$, if $\xi=\varphi(\zeta)$ for some non-decreasing map $\varphi$.

These two notions are equivalent for diffuse random variables $\xi$ and $\zeta$. Clearly $\xi \sim_{c} \zeta$ whenever $\xi / \zeta$. The converse does not hold true, in general. However, $\xi \sim_{c} \zeta$ if and only if $\xi \nearrow \eta$ and $\zeta \nearrow \eta$ for some random variable $\eta$ defined on $(\Omega, \mathcal{F}, \mathbb{P})$. We shall also use the Denneberg's lemma [16] which states that $\xi \sim_{c} \zeta$ if and only if $\xi \nearrow(\xi+\zeta)$ and $\zeta \nearrow(\xi+\zeta)$. We refer to [17] for an overview on these concepts.

Definition 2.3. A function $f: \mathbb{L}^{\infty} \longrightarrow \mathbb{R}$ is said to be comonotone if $f(\xi+\zeta)=f(\xi)+f(\zeta)$ for every pair $(\xi, \zeta)$ of comonotone random variables in $\mathbb{L}^{\infty}$.

Notice that any comonotone function on $\mathbb{L}^{\infty}$ is positively homogeneous. The following result from [32] (see also [25] and [29]) provides a reduction of the quantile representation of Theorem 2.2 to the case of comonotone law-invariant utility functions.

Theorem 2.3 ([32]). Let $U$ be a law-invariant monetary utility function. Then, the following statements are equivalent :

(i) $U$ is comonotone

(ii) there is a (unique) function $\bar{\varphi} \in \overline{\mathcal{C}} \backslash$ such that, with the notations of $(2.3), \operatorname{dom}(v)=$ $\{\varphi \in \mathcal{C} \backslash: \varphi \leq \bar{\varphi}\}, v=0$ on $\operatorname{dom}(v)$, and for every $\xi \in \mathbb{L}^{\infty}$ :

$$
U(\xi)=\int q_{\xi}(t) \bar{\varphi}^{\prime}(t) d t=\inf \left\{\int q_{\xi}(t) \varphi^{\prime}(t) d t: \varphi \in \overline{\mathcal{C}}_{\searrow} \text { and } \varphi \leq \bar{\varphi}\right\} .
$$

Proof. Observe that the set $\{\varphi \in \overline{\mathcal{C}} \backslash: \varphi \leq \bar{\varphi}\}$ is closed and convex. Then, since comonotonicity implies that $U$ is positively homogeneneous and satisfies the Fatou property, see [25] and [29], the proposition is just a restatement from [32].

Example 2.1. Let $\bar{g}_{\alpha}:=\alpha^{-1} \mathbf{1}_{[0, \alpha)}$, and $\bar{\varphi}_{\alpha}(t):=\int_{0}^{t} \bar{g}_{\alpha}(s) d s$, for some $\alpha \in(0,1)$. Then, the corresponding law-invariant comonotone utility function

$$
U_{\alpha}(\xi):=\int q_{\xi}(t) \bar{\varphi}_{\alpha}^{\prime}(t) d t=-\mathrm{AV} @ \mathrm{R}_{\alpha}(\xi)
$$

corresponds to the so-called average value at risk, or expected shortfall, with parameter $\alpha$.

Remark 2.4. Let $U$ be a comonotone law-invariant monetary utility function with associated concave function $\bar{\varphi} \in \overline{\mathcal{C}} \backslash$. Set $\mu:=\bar{\varphi}(0+)$, and $\bar{\varphi}_{*}:=(1-\mu)^{-1}(\bar{\varphi}-\mu)$ on $[0,1]$. Then $\bar{\varphi}_{*} \in \mathcal{C}_{\searrow}$, and

$$
U(\xi)=(1-\mu) U_{*}(\xi)+\mu \text { ess-inf } \xi \quad \text { where } \quad U_{*}(\xi):=\int q_{\xi}(t) \bar{\varphi}_{*}^{\prime}(t) d t .
$$

Hence the possible jump of $\bar{\varphi}$ at the point $t=0$ means that $U(\xi)$ is affected by ess - inf $\xi$. 
We conclude this section by the following explicit calculation of the sup-convolution for two comonotone law-invariant monetary utility functions.

Lemma 2.2. Let $U_{0}$ and $U_{1}$ be two comonotone law-invariant monetary utility functions, and let $\bar{\varphi}_{0}, \bar{\varphi}_{1} \in \overline{\mathcal{C}} \backslash$ be the associated functions in the representation (2.3). Then $U_{0} \square U_{1}$ is a comonotone law-invariant monetary utility function which is associated to $\bar{\varphi}_{0} \wedge \bar{\varphi}_{1} \in \overline{\mathcal{C}} \backslash$, i.e.

$$
U_{0} \square U_{1}(\xi)=\int q_{\xi}(t)\left(\bar{\varphi}_{0} \wedge \bar{\varphi}_{1}\right)^{\prime}(t) d t \quad \text { for every } \quad \xi \in \mathbb{L}^{\infty}
$$

Proof. For a concave function $\varphi:[0,1] \longrightarrow[0,1]$, we introduce the subsets $C_{\varphi}:=$ $\left\{Z \in \mathbb{L}^{1}(\mathbb{P}): \int q_{Z} \in \mathcal{C} \backslash\right.$ and $\left.\int q_{Z} \leq \varphi\right\}$. Observe that $C_{\varphi}$ is closed convex in $\mathbb{L}^{1}(\mathbb{P})$. By Remark 2.3, the functions $U_{i}$ and $V_{i}^{1}=\chi_{C_{\bar{\varphi}_{i}}}$ are $\left\langle\mathbb{L}^{\infty}, \mathbb{L}^{1}(\mathbb{P})\right\rangle$-conjugate, where $\chi$ is the indicator function in the sense of convex analysis. Then, we directly compute that $V_{0}^{1}+V_{1}^{1}=\chi_{C_{\bar{\varphi}_{0}}}+\chi_{C_{\bar{\varphi}_{1}}}=\chi_{C_{\bar{\varphi}_{0}} \cap C_{\bar{\varphi}_{1}}}=\chi_{C_{\bar{\varphi}_{0} \wedge \bar{\varphi}_{1}}}$. The proof is completed by recalling from Remark 2.3 that $U_{0} \square U_{1}$ and $V_{0}^{1}+V_{1}^{1}$ are conjugate.

\section{Optimal risk sharing under law-invariant monetary utility}

\subsection{Pareto optimal allocations}

We consider two agents $i=0,1$. Each agent is characterized by a monetary utility function $U_{i}$, and is endowed with an initial risk $X_{i} \in \mathbb{L}^{\infty}$. The aggregate risk endowment is denoted by

$$
X:=X_{0}+X_{1}
$$

We first recall some classical notions from economic theory, see e.g. Gerber [26]. The affine space

$$
\mathbb{A}(X):=\left\{\left(\xi_{0}, \xi_{1}\right) \in \mathbb{L}^{\infty} \times \mathbb{L}^{\infty}: \xi_{0}+\xi_{1}=X\right\}
$$

is called the set of attainable allocations. This is the collection of all possible sharings of the aggregate risk $X$ between both agents.

Definition 3.1. Let $\left(\xi_{0}, \xi_{1}\right)$ be an attainable allocation in $\mathbb{A}(X)$. We say that $\left(\xi_{0}, \xi_{1}\right)$ is Pareto optimal if for all $\left(\zeta_{0}, \zeta_{1}\right) \in \mathbb{A}(X)$ :

$$
U_{0}\left(\zeta_{0}\right) \geq U_{0}\left(\xi_{0}\right) \text { and } U_{1}\left(\zeta_{1}\right) \geq U_{1}\left(\xi_{1}\right) \Longrightarrow U_{0}\left(\zeta_{0}\right)=U_{0}\left(\xi_{0}\right) \text { and } U_{1}\left(\zeta_{1}\right)=U_{1}\left(\xi_{1}\right) \text {. }
$$

The well-known concept of Pareto optimal allocations describes the possible candidates for an optimal way of risk sharing. Indeed if $\left(\xi_{0}, \xi_{1}\right)$ fails to be Pareto-optimal, one may find a pair $\left(\zeta_{0}, \zeta_{1}\right)$ such that both agents are better of, and at least one of them is strictly better. 
Remark 3.1. In the context of monetary utility functions, notice that

1. Pareto optimal allocations are only defined up to a constant, i.e. given a Pareto optimal allocation $\left(\xi_{0}, \xi_{1}\right)$, the attainable allocation $\left(\xi_{0}+c, \xi_{1}-c\right)$ is also Pareto optimal for every $c \in \mathbb{R}$,

2. Let $\left(\xi_{0}, \xi_{1}\right)$ be an attainable allocation which is not Pareto optimal. Then, we can always find a Pareto optimal allocation $\left(\zeta_{0}, \zeta_{1}\right)$ such that $U_{0}\left(\zeta_{0}\right)>U_{0}\left(\xi_{0}\right)$ and $U_{1}\left(\zeta_{1}\right)>U_{1}\left(\xi_{1}\right)$; this follows immediately from the cash invariance of $U_{0}$ and $U_{1}$.

The key-ingredient for the characterization of Pareto optimal allocations is the following.

Theorem 3.1. Let $U_{0}$ and $U_{1}$ be two monetary utility functions with associated convex conjugate functions $V_{0}$ and $V_{1}$ defined on $\left(\mathbb{L}^{\infty}\right)^{*}$. For a given aggregate risk $X \in \mathbb{L}^{\infty}$ and $\left(\xi_{0}, \xi_{1}\right) \in \mathbb{A}(X)$, the following statements are equivalent :

(i) $\left(\xi_{0}, \xi_{1}\right)$ is a Pareto optimal allocation,

(ii) $U_{0} \square U_{1}(X)=U_{0}\left(\xi_{0}\right)+U_{1}\left(\xi_{1}\right)$,

(iii) $U_{i}\left(\xi_{i}\right)=V_{i}(\mu)+\left\langle\mu, \xi_{i}\right\rangle, i=0,1$, for some $\mu \in\left(\mathbb{L}^{\infty}\right)^{*}$,

(iv) $-\xi_{i} \in \partial V_{i}(\mu), i=0,1$, for some $\mu \in\left(\mathbb{L}^{\infty}\right)^{*}$,

(v) $\partial U_{0}\left(\xi_{0}\right) \cap \partial U_{1}\left(\xi_{1}\right) \neq \emptyset$.

If $U_{0}$ and $U_{1}$ are in addition law-invariant, then any of the above assertions holds iff (vi) $\left(\bar{\xi}_{0}, \bar{\xi}_{1}\right):=\left(\mathbb{E}\left[\xi_{0} \mid X\right], \mathbb{E}\left[\xi_{1} \mid X\right]\right) \in \mathbb{A}(X)$ is a Pareto optimal allocation and $U_{i}\left(\bar{\xi}_{i}\right)=$ $U_{i}\left(\xi_{i}\right)$, for $i=0,1$.

Proof. The equivalences (ii)-(v) are known duality results, even in a more general dynamic context, see Klöppel and Schweizer [31]. The connection with (i) is a classical result in economic theory, see e.g. [13]. Equivalence with (vi) in the law-invariant context is new. For completeness, we report the proof of all equivalences.

(iii) $\Longleftrightarrow$ (iv) $\Longleftrightarrow$ (v) hold true by definition of the convex conjugate, and the Fenchel duality relation.

(ii) $\Longleftrightarrow$ (iii) Let $\mu \in \partial\left(U_{0} \square U_{1}\right)(X)$. Following the argument of Theorem 10 in [6], we compute that

$$
\begin{aligned}
U_{0} \square U_{1}(X) & =\langle\mu, X\rangle+V_{0}(\mu)+V_{1}(\mu) \\
& =\langle\mu, X\rangle+\sup _{\xi \in \mathbb{L}^{\infty}}\left(U_{0}(\xi)-\langle\mu, \xi\rangle\right)+\sup _{\xi \in \mathbb{L}^{\infty}}\left(U_{1}(X-\xi)-\langle\mu, X-\xi\rangle\right) \\
& =\sup _{\xi \in \mathbb{L}^{\infty}}\left(U_{0}(\xi)-\langle\mu, \xi\rangle\right)+\sup _{\xi \in \mathbb{L}^{\infty}}\left(U_{1}(X-\xi)+\langle\mu, \xi\rangle\right) \\
& \geq \sup _{\xi \in \mathbb{L}^{\infty}}\left(U_{0}(\xi)+U_{1}(X-\xi)\right)=U_{0} \square U_{1}(X) .
\end{aligned}
$$

Hence $U_{0} \square U_{1}(X)=U_{0}\left(\xi_{0}\right)+U_{1}\left(X-\xi_{0}\right)$ if and only if $V_{0}(\mu)=U_{0}\left(\xi_{0}\right)-\left\langle\mu, \xi_{0}\right\rangle$ and $V_{1}(\mu)=U_{1}\left(X-\xi_{0}\right)-\left\langle\mu, X-\xi_{0}\right\rangle$.

(ii) $\Longrightarrow$ (i) is trivial, and (i) $\Longrightarrow$ (ii) Let $\left(\xi_{0}, \xi_{1}\right)$ be a Pareto optimal allocation, and consider the sets $\tilde{B}:=\left\{\left(U_{0}\left(\zeta_{0}\right), U_{1}\left(\zeta_{1}\right)\right):\left(\zeta_{0}, \zeta_{1}\right) \in \mathbb{A}(X)\right\}, B:=\tilde{B}-\mathbb{R}_{-}^{2}$, and $C:=$ 
$\left\{\left(U_{0}\left(\xi_{0}\right), U_{1}\left(\xi_{1}\right)\right)\right\}+\left(\mathbb{R}_{+}^{2} \backslash\{0\}\right)$. It is immediately checked that $B$ and $C$ are non-empty convex subsets of $\mathbb{R}^{2}$ with non-empty interior, and $B \cap C=\emptyset$ by the Pareto optimality of $\left(\xi_{0}, \xi_{1}\right)$. By the Hahn-Banach separation Theorem, there exists $\lambda \in \mathbb{R}^{2} \backslash\{0\}$ such that $\lambda \cdot y \leq \lambda \cdot z$ for all $(y, z) \in B \times C$. Since $\left(U_{0}\left(\xi_{0}\right), U_{1}\left(\xi_{1}\right)\right) \in B,\left(U_{0}\left(\xi_{0}\right)+1, U_{1}\left(\xi_{1}\right)\right) \in C$ and $\left(U_{0}\left(\xi_{0}\right), U_{1}\left(\xi_{1}\right)+1\right) \in C$, we deduce from the separation inequality that $\lambda_{i} \geq 0$ for $i=0,1$. It then follows from the separation inequality that $\left(\xi_{0}, \xi_{1}\right)$ is a maximizer of $\lambda_{0} U_{0}\left(\zeta_{0}\right)+\lambda_{1} U_{1}\left(\zeta_{1}\right)$ over $\left(\zeta_{0}, \zeta_{1}\right) \in \mathbb{A}(X)$. Finally, the cash-invariance properties of $U_{0}$ and $U_{1}$ imply that $\lambda_{0}=\lambda_{1}$.

(vi) $\Longrightarrow$ (i) is trivial. To see that (i) $\Longrightarrow$ (vi) for comonotone $U_{0}, U_{1}$, we recall from Lemma 3.2 in $[29]$ that $\mathbb{E}\left[\xi_{i} \mid X\right]$ lies in the $\sigma\left(\left(\mathbb{L}^{\infty}\right)^{*}, \mathbb{L}^{\infty}\right)$-closed convex hull of the set $\left\{\zeta \in \mathbb{L}^{\infty}: \zeta={ }_{d} \xi_{i}\right\}$. Then, it follows from the concavity and the law-invariance of $U_{i}$ that $U_{i}\left(\mathbb{E}\left[\xi_{i} \mid X\right]\right) \geq U_{i}\left(\xi_{i}\right)$.

The characterization (ii) in the above Theorem 3.1 has a well-known extension for general utility functions (see e.g. [26]), which involves two Lagrange multipliers (simply leaving out the last sentence in the above proof of $(\mathrm{i}) \Longrightarrow(\mathrm{ii}))$. In the general case, the Pareto optimal allocations are characterized as the pairs $\left(\xi_{0}^{*}(\lambda), \xi_{1}^{*}(\lambda)\right)$ such that $\left(\lambda_{0} U_{0}\right) \square\left(\lambda_{1} U_{1}\right)(X)=$ $\lambda_{0} U_{0}\left(\xi_{0}(\lambda)\right)+\lambda_{1} U_{1}\left(\xi_{1}^{*}(\lambda)\right)$, for some $\lambda=\left(\lambda_{0}, \lambda_{1}\right) \in \mathbb{R}_{+}^{2} \backslash\{0\}$. It is remarkable that the cash-invariance property reduces the set of such Lagrange multipliers to $\lambda_{0}=\lambda_{1}$, and leads to the sup-convolution $U_{0} \square U_{1}$.

Remark 3.2. The equivalences (ii) $\Longleftrightarrow($ iii $) \Longleftrightarrow($ iv $) \Longleftrightarrow(v)$ in Theorem 3.1 hold true for general concave proper functions $U_{0}$ and $U_{1}$. The monotonicity and the cash invariant properties assumed in the statement of the theorem are only needed in order to connect these properties to (i).

\subsection{The existence result}

Let $\mathbb{A} \nearrow(X):=\left\{\left(\xi_{0}, \xi_{1}\right) \in \mathbb{A}(X): \xi_{0} \nearrow X\right.$ and $\left.\xi_{1} \nearrow X\right\}$ be the subset of admissible allocations which increase with the corresponding aggregate risk. By the Denneberg's lemma [16], we observe that $\mathbb{A} \nearrow(X)$ is the subset of $\mathbb{A}(X)$ consisting of all comonotone allocations. The following result is proved in Section 6 , where we also discuss the fact that the law-invariance assumption can not be dropped.

Theorem 3.2. Let $U_{0}$ and $U_{1}$ be two law-invariant monetary utility functions. Then for every $X \in \mathbb{L}^{\infty}, U_{0} \square U_{1}(X)=\sup _{\left(\xi_{0}, \xi_{1}\right) \in \mathbb{A} \nearrow(X)} U_{0}\left(\xi_{0}\right)+U_{1}\left(\xi_{1}\right)$, and the set of Pareto optimal allocations in $\mathbb{A} \nearrow(X)$ is non-empty.

Recall from Theorem 3.1 (vi) that any Pareto optimal allocation $\left(\xi_{0}, \xi_{1}\right)$ induces a Pareto optimal allocation $\left(\bar{\xi}_{0}, \bar{\xi}_{1}\right)=\left(\mathbb{E}\left[\xi_{0} \mid X\right], \mathbb{E}\left[\xi_{1} \mid X\right]\right)$ which is a measurable function of $X$. The above existence result asserts that one may find such Pareto optimal allocations which 
increase in $X$. In general, there is no hope for any uniqueness of Pareto optimal allocations in this class. To illustrate the situation, we consider a rather trivial example : let $U_{i}(X)=$ $\mathbb{E}[X]$ for $i=0,1$, and consider $X=0$. Then any $\left(\xi_{0}, \xi_{1}\right) \in \mathbb{A}(X)$ is Pareto-optimal.

\subsection{Two concrete examples}

After having established the existence of Pareto optimal allocations, we now give an explicit characterization of Pareto optimal allocations in $\mathbb{A} \nearrow(X)$ for some specific lawinvariant monetary utility functions. We first consider the case where both agents are defined by comonotone law-invariant monetary utility functions.

Definition 3.2. Let $(\varphi, \psi)$ be a pair of functions in $\overline{\mathcal{C}} \backslash$ with $\varphi \leq \psi$, and $f$ a nondecreasing function on $[0,1]$. We say that $f$ is flat on $\{\varphi<\psi\}$ if $d f=0$ a.e. on $\{\varphi<\psi\}$ and $(f(0+)-f(0))(\varphi-\psi)(0+)=0$.

Proposition 3.1. Assume that the preferences of each agent $i=0,1$ are characterized by a comonotone law-invariant monetary utility function, and let $\bar{\varphi}_{i} \in \overline{\mathcal{C}}_{\searrow}$ be the corresponding non-decreasing concave function, as defined in Theorem 2.3. Let $X \in \mathbb{L}^{\infty}$ be some given aggregate risk endowment. For $\left(\xi_{0}, \xi_{1}\right) \in \mathbb{A} \nearrow(X)$, the following statements are equivalent :

(i) $\left(\xi_{0}, \xi_{1}\right)$ is a Pareto optimal allocation,

(ii) the quantile function $q_{\xi_{i}}$ is flat on $\left\{\bar{\varphi}_{i}>\bar{\varphi}_{1-i}\right\} \cap\left\{d q_{X}>0\right\}$.

The proof of Proposition 3.1 is reported in Section 4. In the context of this result, we observe that, for a Pareto optimal allocation $\left(\xi_{0}, \xi_{1}\right) \in \mathbb{A}^{\nearrow}(X)$, we have in addition that $q_{\xi_{i}}$ is flat on $\left\{\bar{\varphi}_{0} \neq \bar{\varphi}_{1}\right\} \cap\left\{d q_{X}=0\right\}$, for $i=0,1$.

The following easy application relates this result to options or stop-loss contracts, layers and deductibles in insurance.

Example 3.1. Let $N_{0}, N_{1}$ be two given integers, and consider the utility functions $U_{i}(X):=$ $-\sum_{j=1}^{N_{i}} \mu_{i}^{j} \mathrm{AV} @ \mathrm{R}_{\alpha_{i}^{j}}(X)$, where $\mu_{i}^{j}>0, \sum_{i=1}^{N_{i}} \mu_{i}^{j}=1,\left(\alpha_{i}^{j}, 1 \leq j \leq N_{i}\right) \in(0,1)^{N_{i}}, i=0,1$. Then the Pareto optimal allocations of Proposition 3.1 correspond to a finite sum of European options on the total risk $X$, i.e.

$$
\xi_{0}:=\sum_{k=1}^{K} \beta_{k}\left(X-c_{k}\right)^{+} \quad \text { and } \quad \xi_{1}:=X-\xi_{0},
$$

for some integer $K$ and $\left(\beta_{k}, 1 \leq k \leq K\right) \in \mathbb{R}^{K}$.

We next restrict the preferences of Agent 0 to the prime example of comonotone lawinvariant monetary utility function :

$$
U_{0}(X):=-\mathrm{AV} @ \mathrm{R}_{\alpha}(X), \quad X \in \mathbb{L}^{\infty}, \text { for some fixed } \alpha \in(0,1),
$$


where $\mathrm{AV} @ \mathrm{R}_{\alpha}$ is the average value at risk defined in Example 2.1. As for Agent 1, we consider a rather general class of law-invariant monetary utility functions $U_{1}$. We shall restrict agent 1 to have a strictly monotone utility function, i.e. $U_{1}(\xi)>U_{1}(\zeta)$ whenever $\xi-\zeta \in \mathbb{L}_{+}^{\infty} \backslash\{0\}$, with a strict risk-aversion property to be defined below. Before turning to the precise definition of the latter restriction, we state our second main concrete example.

Proposition 3.2. Let $U_{0}$ be given by (3.1), and let $U_{1}$ be a law-invariant monetary utility function. Assume that $U_{1}$ is strictly monotone and strictly risk averse conditionally on lower tail events. Let $X \in \mathbb{L}^{\infty}$ be the aggregate risk endowment. Then there is a unique (up to a constant) Pareto optimal allocation in $\mathbb{A}(X)$ defined by

$$
\left(\xi_{0}, \xi_{1}\right):=\left(-(X-\kappa)^{-}, X \vee \kappa\right)
$$

for some $\kappa \in \mathbb{R}$.

The proof of Proposition 3.2 is reported in Section 5. We now explain the notion of risk aversion involved in its statement.

Definition 3.3. (i) Let $\xi \in \mathbb{L}^{\infty}$ and $A \in \mathcal{F}$ with $\mathbb{P}[A]>0$. We say that $A$ is a lower tail-event for $\xi$, and we denote $A \in \mathcal{F}^{\downarrow}(\xi)$, if

$$
\operatorname{ess}^{-}-\inf _{A} \xi<\operatorname{ess}-\sup _{A} \xi \leq \operatorname{ess}^{-} \inf _{A^{c}} \xi .
$$

(ii) Let $U$ be a monetary utility function. We say that $U$ is strictly risk-averse conditionally on lower tail-events if

$$
U(\xi)<U\left(\xi \mathbf{1}_{A^{c}}+\mathbb{E}[\xi \mid A] \mathbf{1}_{A}\right) \quad \text { for every } \quad \xi \in \mathbb{L}^{\infty} \text { and } A \in \mathcal{F}^{\downarrow}(\xi) .
$$

As a matter of illustration, we provide a characterization of the notions of strict monotonicity and strict risk aversion conditionally on lower tail events in the context of comonotone law-invariant monetary utility functions. In particular, Lemma 3.1 below, whose proof is given in Appendix 1, shows that none of these properties is satisfied by the utility function $U_{0}=-\mathrm{AV} @ \mathrm{R}_{\alpha}$.

Lemma 3.1. Let $U$ be a comonotone law-invariant monetary utility function, with associated non-decreasing concave function $\bar{\varphi} \in \overline{\mathcal{C}} \searrow$, i.e. $U(\xi)=\int_{0}^{1} q_{\xi}(t) \bar{\varphi}^{\prime}(t) d t$ for every $\xi \in \mathbb{L}^{\infty}$, see Theorem 2.3. Then :

(i) $U$ is strictly risk averse conditionally on lower tail events if and only if $\bar{\varphi}$ is not linear near the origin, i.e. there does not exist $c>0$ and $\varepsilon>0$ such that $\bar{\varphi}(t)=c t$ for $0 \leq t \leq \varepsilon$.

(ii) $U$ is strictly monotone if and only if $\bar{\varphi}(t)<1$ for $0 \leq t<1$. 


\subsection{The semi-deviation utility}

In this section, we exemplify the previous notions by considering the semi-deviation utility function :

$$
U_{1}(\xi):=\mathbb{E} \xi-\delta \mathbb{E}\left[(\xi-\mathbb{E} \xi)_{-}^{p}\right]^{1 / p} \quad \text { where } \quad 0 \leq \delta \leq 1 \text { and } 1 \leq p \leq \infty,
$$

compare e.g. [23]. Our purpose is to verify that Proposition 3.2 applies to this example for $1<p<\infty$.

(i) The function $U_{1}$ of (3.2) defines a positively homogeneous law-invariant monetary utility function. Clearly, $U_{1}$ is comonotone for $p=\infty$. Observe however that $U_{1}$ is not comonotone for $p<\infty$. For instance, this can be easily checked in the following example. Let $(\Omega, \mathcal{F}, \mathbb{P})=\{[0,1], \mathcal{B}, \lambda\}$, and consider the comonotone random variables $f(t)=t$, $g(t)=t^{2}$ for every $t \in[0,1]$. Direct computation shows that $U_{1}(f+g) \neq U_{1}(f)+U_{1}(g)$ for $p<\infty$.

(ii) We now check that the semi-deviation utility function $U_{1}$ is strictly risk averse conditionally on lower tail events for $1<p \leq \infty$. To see this, let $\xi \in \mathbb{L}^{\infty}, A \in \mathcal{F} \downarrow(\xi)$, and set $\tilde{\xi}$ $:=\xi \mathbf{1}_{A^{c}}+\mathbb{E}[\xi \mid A] \mathbf{1}_{A}$. Since $\mathbb{E} \tilde{\xi}=\mathbb{E} \xi$, we have

$$
\begin{aligned}
U_{1}(\tilde{\xi}) & =\mathbb{E} \xi-\delta \mathbb{E}\left[(\tilde{\xi}-\mathbb{E} \xi)_{-}^{p}\right]^{1 / p} \\
& =\mathbb{E} \xi-\delta\left\{\mathbb{E}\left[(\xi-\mathbb{E} \xi)_{-}^{p} \mathbf{1}_{A^{c}}\right]+\mathbb{E}\left[(\mathbb{E}[\xi-\mathbb{E} \xi \mid A])_{-}^{p} \mathbf{1}_{A}\right]\right\}^{1 / p} .
\end{aligned}
$$

Since $z \longmapsto z_{-}^{p}$ is strictly convex on $\mathbb{R}_{-}$for $1<p<\infty$, and $(\xi-\mathbb{E} \xi)_{-} \mathbf{1}_{A}$ is not a.s. constant, it follows from the Jensen inequality that

$$
U_{1}(\tilde{\xi})>\mathbb{E} \xi-\delta\left\{\mathbb{E}\left[(\xi-\mathbb{E} \xi)_{-}^{p} \mathbf{1}_{A^{c}}\right]+\mathbb{E}\left[\mathbb{E}\left[(\xi-\mathbb{E} \xi)_{-}^{p} \mid A\right] \mathbf{1}_{A}\right]\right\}^{1 / p}=U_{1}(\xi) .
$$

The above strict inequality is still valid for the case $p=\infty$. However, for $p=1$, the semi-deviation utility is not strictly risk averse conditionally on lower tail events. Indeed, the last strict inequality is turned into an equality for $A:=\{\xi-\mathbb{E} \xi \leq 0\} \in \mathcal{F} \downarrow(\xi)$.

(iii) Finally, it is easy to check that the semi-deviation utility function $U_{1}$ of $(3.2)$ is strictly monotone, except for the case $p=\infty, \delta=1$.

\subsection{The entropic utility}

We now show that the result of Proposition 3.2 applies to the popular entropic utility example. For a fixed constant $\eta>0$, let

$$
U_{1}(\xi):=-\frac{1}{\eta} \ln \mathbb{E}\left[e^{-\eta \xi}\right] \quad \text { for } \quad \xi \in \mathbb{L}^{\infty} .
$$


Clearly $U_{1}$ is strictly monotone. We now show that $U_{1}$ is strictly risk-averse conditionally on any event $A \in \mathcal{F}$ such that $\xi$ is not a.s. constant on $A$. Indeed, for $\xi \in \mathbb{L}^{\infty}$ and $\bar{\xi}:=\xi \mathbf{1}_{A^{c}}+\mathbb{E}[\xi \mid A] \mathbf{1}_{A}$, it follows from the strict convexity of the exponential together with the Jensen inequality applied to the random variable $\xi \mathbf{1}_{A}$ that

$$
\mathbb{E}\left[\mathbf{1}_{A} e^{-\eta \bar{\xi}}\right]<\mathbb{E}\left[\mathbf{1}_{A} e^{-\eta \xi}\right]
$$

Hence

$$
\begin{aligned}
U_{1}(\bar{\xi}) & =-\frac{1}{\eta} \ln \mathbb{E}\left[e^{-\eta \bar{\xi}}\left(\mathbf{1}_{A}+\mathbf{1}_{A^{c}}\right)\right] \\
& >-\frac{1}{\eta} \ln \mathbb{E}\left[e^{-\eta \xi}\left(\mathbf{1}_{A}+\mathbf{1}_{A^{c}}\right)\right]=U_{1}(\xi) .
\end{aligned}
$$

\subsection{Optimal risk sharing allocations}

Definition 3.4. Let $\left(\xi_{0}, \xi_{1}\right)$ be an attainable allocation in $\mathbb{A}(X)$. We say that $\left(\xi_{0}, \xi_{1}\right)$ is an optimal risk sharing rule if it is Pareto optimal and satisfies the individual rationality constraint (IR) : $U_{i}\left(\xi_{i}\right) \geq U_{i}\left(X_{i}\right)$ for $i=0,1$.

In the framework of the paradigm of rational expectations, an attainable allocation which does not satisfy the IR constraint is rejected by one of the two agents, as the exchange of risk according to such an allocation strictly decreases her utility.

By definition, any optimal risk sharing rule is a Pareto optimal allocation. The main goal of this section is to show that a Pareto optimal allocation induces an optimal risk sharing rule by associating to it a suitable price. Consequently, the explicit Pareto optimal allocations obtained in Propositions 3.1 and 3.2 induce optimal risk sharing rules.

We first introduce the following maps from $\mathbb{L}^{\infty}$ to $\mathbb{R}$ :

$$
p_{0}(\xi):=U_{0}\left(X_{0}\right)-U_{0}\left(X_{0}-\xi\right) \text { and } p_{1}(\xi):=U_{1}\left(X_{1}+\xi\right)-U_{1}\left(X_{1}\right) \text {, }
$$

which are known as the reservation prices of the agents. Here, $p_{0}(\xi)$ is the minimal price for which Agent 0 is willing to transfer the amount of wealth $\xi$ to Agent 1 , and $p_{1}(\xi)$ is the maximal price for which Agent 1 is willing to accept receiving the amount of wealth $\xi$ from Agent 1. Obviously, under the condition (IR) of individual rationality, the agents can agree to exchange the risk $\xi$ if and only if $p_{0}(\xi) \leq p_{1}(\xi)$. In the latter case, for every $p \in\left[p_{0}(\xi), p_{1}(\xi)\right]$, the allocation $\left(X_{0}-(\xi-p), X_{1}+(\xi-p)\right) \in \mathbb{A}(X)$ satisfies the individual rationality condition (IR).

Theorem 3.3. Let $U_{0}$ and $U_{1}$ be two monetary utility functions, and let $\left(X_{0}-\xi^{*}, X_{1}+\xi^{*}\right)$ be a Pareto optimal allocation. Then $p_{0}\left(\xi^{*}\right) \leq p_{1}\left(\xi^{*}\right)$, and $\left(X_{0}-\xi^{*}+p, X_{1}+\xi^{*}-p\right)$ is an optimal risk sharing allocation if and only if the real number $p$ lies in $\left[p_{0}\left(\xi^{*}\right), p_{1}\left(\xi^{*}\right)\right]$. 
Proof. In view of the discussion preceding the theorem, we only need to show that $p_{0}\left(\xi^{*}\right) \leq p_{1}\left(\xi^{*}\right)$. This follows from Theorem 3.1, as

$$
p_{0}\left(\xi^{*}\right)-p_{1}\left(\xi^{*}\right)=\left[U_{0}\left(X_{0}\right)+U_{1}\left(X_{1}\right)\right]-\left[U_{0}\left(X_{0}-\xi^{*}\right)+U_{1}\left(X_{1}+\xi^{*}\right)\right] \leq 0 .
$$

Theorem 3.3 shows that, when both agents have monetary utility functions, the determination of an optimal risk sharing allocation reduces to the characterization of a Pareto optimal allocation $\left(X_{0}-\xi^{*}, X_{1}+\xi^{*}\right)$ in $\mathbb{A}(X)$ (which does exist in the law invariant case by Theorem 3.2). The width

$$
w:=p_{1}\left(\xi^{*}\right)-p_{0}\left(\xi^{*}\right)=U_{0} \square U_{1}(X)-U_{0}\left(X_{0}\right)-U_{1}\left(X_{1}\right)
$$

may be called the rent of risk exchange. Observe that $w$ depends only on $X$ and not on the choice of the Pareto optimal allocation $\left.\left(X_{0}-\xi^{*}, X_{1}+\xi^{*}\right)\right)$. Given $p \in\left[p_{0}\left(\xi^{*}\right), p_{1}\left(\xi^{*}\right)\right]$, this quantity clearly decomposes into $w=w_{0}+w_{1}$, where $w_{i}$ is the utility gain of agent $i$ from the risk exchange defined by

$$
w_{i}:=U_{i}\left(\xi_{i}\right)-U_{i}\left(X_{i}\right), \quad \xi_{0}:=X_{0}-\left(\xi^{*}-p\right), \quad \xi_{1}:=X_{0}+\left(\xi^{*}-p\right) .
$$

In the special case $w=0$, the initial risk endowment $\left(X_{0}, X_{1}\right)$ is an optimal risk sharing allocation. In this case, it is optimal not to exchange any amount of risk.

If $w>0$, i.e. $p_{0}\left(\xi^{*}\right)<p_{1}\left(\xi^{*}\right)$, then the exchange of the amount of risk $\xi^{*}$ is possible between Agents 0 and 1 , and the choice of the price $p \in\left[p_{0}\left(\xi^{*}\right), p_{1}\left(\xi^{*}\right)\right]$ corresponding to this transaction depends on the market power of the agents :

(i) If Agent 0 has the power to design the contract and to impose it as a take-it-or-leaveit proposal to Agent 1, then Agent 0 will impose the largest price $p=p_{1}\left(\xi^{*}\right)$ so that the utility of Agent 1 is not decreased by the exchange, i.e. $U_{1}\left(X_{1}+\xi^{*}-p\right)=U_{1}\left(X_{1}\right)$. This is the point of view of Barrieu and ElKaroui $[3,4,5,6]$. In practice, Agent 1 then has no incentive to proceed to such a risk exchange as her utility is not altered. Since Agent 0 is interested in the exchange, she should rather propose the price $p_{\varepsilon}=p_{1}\left(\xi^{*}\right)-\varepsilon w$ for some small parameter $\varepsilon>0$, so that the utility of Agent 1 is strictly increased by the exchange in order to give some incentive to agent 1.

(ii) In contrast to the preceding argument relying on the paradigm of rational expectations, the behavioral viewpoint tells us a very different story about the parameter $\varepsilon>0$ introduced above. For example, the experiments on the ultimatum game reveal that Agent 0 should choose the parameter $\varepsilon$ above of the order of 0.3 or 0.4 . See [11], [30], and [35] for further details on this issue. Of course, the value of $\varepsilon$ depends strongly on the design of these experiments, and the real-life situation to which the present model is applied. The ultimatum game is a rather different situation than the setting covered by the subsequent examples of this paper, where it seems appropriate to expect a more rational behavior of the agents. 


\section{Super-gradient of law-invariant monetary utility functions}

The main purpose of this section is to prove Proposition 3.1. By Theorem 3.1, Pareto optimal allocations are the solutions of the optimization problem $U_{0} \square U_{1}$, and are characterized in terms of the super-gradient of the concave functions $U_{i}$ for $i=0,1$.

We first need to extend the notion of law-invariance for functions defined on $\left(\mathbb{L}^{\infty}\right)^{*}$. A measure preserving transformation of $(\Omega, \mathcal{F}, \mathbb{P})$ is a bi-measurable bijection $\tau:(\Omega, \mathcal{F}, \mathbb{P}) \rightarrow$ $(\Omega, \mathcal{F}, \mathbb{P})$ leaving $\mathbb{P}$ invariant, i.e., $\tau(\mathbb{P})=\mathbb{P}$. The transformation $\tau$ induces an isometric isomorphism on $\mathbb{L}^{\infty}(\mathbb{P})$, still denoted by $\tau$, defined by $\tau(\xi)=\xi \circ \tau$ for every $\xi \in \mathbb{L}^{\infty}$. The transpose of $\tau: \mathbb{L}^{\infty} \rightarrow \mathbb{L}^{\infty}$, denoted by $\tau^{*}$, defines an isometry on $\left(\mathbb{L}^{\infty}\right)^{*}$ via

$$
\left\langle\tau^{*}(\mu), \xi\right\rangle=\langle\mu, \tau(\xi)\rangle \text { for every } \mu \in\left(\mathbb{L}^{\infty}\right)^{*} \text { and } \xi \in \mathbb{L}^{\infty} .
$$

A function $f:\left(\mathbb{L}^{\infty}\right)^{*} \longrightarrow \mathbb{R}$ is called law invariant if $f=f \circ \tau^{*}$ for every measure preserving transformation $\tau:(\Omega, \mathcal{F}, \mathbb{P}) \rightarrow(\Omega, \mathcal{F}, \mathbb{P})$.

The statement of our first result requires to extend the notion of comonotonicity to bounded finitely additive measures.

Definition 4.1. Let $\xi$ be a random variable with $\xi^{+} \in \mathbb{L}^{\infty}$, and $\mu \in\left(\mathbb{L}^{\infty}\right)^{*}$ with (unique) decomposition $\mu=\mu^{r}+\mu^{s}$ into a countably additive measure $\mu^{r}=Z \cdot \mathbb{P}$ (regular part) and a purely finitely additive measure $\mu^{s}$ (singular part). We say that $\mu$ is comonotone to (resp. increases with) $\xi$, and we write $\mu \sim_{c} \xi$ (resp. $\mu \nearrow \xi$ ), if

(i) $Z$ is comonotone to (resp. increase with) $\xi$, and

(ii) $\mu^{s}$ is concentrated on $\{\xi=\operatorname{ess}-\sup \xi\}$ in the sense that $\mu^{s}[\{\xi>\operatorname{ess}-\sup \xi-\varepsilon\}]=$ $\left\|\mu^{s}\right\|_{\text {ba }}$ for every $\varepsilon>0$.

We start by considering law-invariant monetary utility functions, before specializing the discussion to the comonotone case.

Lemma 4.1. Let $U$ be a law-invariant monetary utility function, $\xi \in \mathbb{L}^{\infty}$, and let $\mu \in$ $\left(\mathbb{L}^{\infty}\right)^{*}$ be in $\partial U(\xi)$. Then

(i) $-\mu \sim_{c} \xi$,

(ii) $\mathbb{E}[\mu \mid \mathcal{G}] \in \partial U(\xi)$ for any sub-sigma-algebra $\mathcal{G}$ of $\mathcal{F}$ with $\sigma(\xi) \subset \mathcal{G}$.

In particular, $-\mathbb{E}[\mu \mid \xi] \nearrow \xi$ and $\mathbb{E}[Z \mid \xi] \in \partial U(\xi)$.

Proof. Fix $\mu \in \partial U(\xi)$ with decomposition $\mu=\mu^{r}+\mu^{s}$ into its regular part $\mu^{r}$ and its singular part $\mu^{s}$. By definition of the supergradient, we have $U(\xi)=\langle\mu, \xi\rangle+V(\mu)=$ $\inf \left\{\langle\nu, \xi\rangle+V(\nu): \nu \in\left(\mathbb{L}^{\infty}\right)^{*}\right\}$.

1. We first prove that $-\mu^{s}$ is concentrated on $\{\xi=$ ess-sup $\xi\}$ in the sense of Definition 4.1. To do this, we assume to the contrary that $-\mu^{s}$ is not concentrated on 
$\{\xi=$ ess- sup $\xi\}$, and we construct a measure preserving transformation $\tau:(\Omega, \mathcal{F}, \mathbb{P}) \longrightarrow$ $(\Omega, \mathcal{F}, \mathbb{P})$ such that

$$
\mu_{1}:=\mu \circ \tau^{*} \text { satisfies }\left\langle\mu_{1}, \xi\right\rangle<\langle\mu, \xi\rangle \text {. }
$$

By the law-invariance of $V$ (inherited from $U$ ), this implies that $U(\xi)=\langle\mu, \xi\rangle+V(\mu)\rangle$ $\left\langle\mu_{1}, \xi\right\rangle+V(\mu)=\left\langle\mu_{1}, \xi\right\rangle+V\left(\mu_{1}\right) \geq U(\xi)$, a contradiction.

We shall denote $A_{\gamma}:=\{\xi<$ ess-inf $\xi+\gamma\}$ for every $\gamma>0$. If $-\mu^{s}$ is not concentrated on $\{\xi=\operatorname{ess}-\sup \xi\}$, then

$$
\alpha:=\mu^{s}\left[\Omega \backslash A_{2 \varepsilon}\right]>0 \text { for some } \varepsilon>0,
$$

and we may find two sequences $\left(B_{n}\right)_{n \geq 1} \subset\left(\Omega \backslash A_{2 \varepsilon}\right)$ and $\left(C_{n}\right)_{n \geq 1} \subset A_{\varepsilon}$ such that

$$
\mathbb{P}\left[B_{n}\right]=\mathbb{P}\left[C_{n}\right] \leq \frac{1}{n} \quad \text { while } \mu^{s}\left[B_{n}\right]=\alpha \text { and } \mu^{s}\left[C_{n}\right]=0 .
$$

Next, let $\tau_{n}:(\Omega, \mathcal{F}, \mathbb{P}) \longrightarrow(\Omega, \mathcal{F}, \mathbb{P})$ be a bijective measure preserving transformation such that $\tau_{n}\left(B_{n}\right)=C_{n}, \tau_{n}\left(C_{n}\right)=B_{n}$, and $\tau_{n}=I d$ on $\Omega \backslash\left(B_{n} \cup C_{n}\right)$. Set $\nu_{n}:=\mu \circ \tau_{n}^{*}$, $\bar{\mu}:=\mu^{s} \mathbf{1}_{\Omega \backslash A_{2 \varepsilon}}, \bar{\mu}_{n}:=\bar{\mu} \circ \tau_{n}^{*}$. Observing that $\|\bar{\mu}\|_{\text {ba }}=\alpha$, we directly estimate that

$$
\begin{aligned}
\lim _{n \rightarrow \infty}\left\langle\mu-\nu_{n}, \xi\right\rangle & =\lim _{n \rightarrow \infty}\left\langle\bar{\mu}-\bar{\mu}_{n}, \xi\right\rangle+\lim _{n \rightarrow \infty}\left\langle\left(\mu^{r}-\mu_{n}^{r}\right) \mathbf{1}_{B_{n} \cup C_{n}}, \xi\right\rangle \\
& =\lim _{n \rightarrow \infty}\left\langle\bar{\mu}-\bar{\mu}_{n}, \xi\right\rangle \leq \alpha(\varepsilon-2 \varepsilon)<0,
\end{aligned}
$$

so that $\mu_{1}:=\nu_{n}$, for a sufficiently large integer $n$, satisfies (4.1).

2. Let $\mathcal{G}$ be any sub- $\sigma$-algebra of $\mathcal{F}$. Since $\mu \in \operatorname{dom}(V)$, it follows from Lemma 3.3 in $[29]$ that $\mu_{1}:=\mathbb{E}[\mu \mid \mathcal{G}] \in \operatorname{dom}(V)$, and $V(\mu) \geq V\left(\mu_{1}\right)$ by the convexity and the $\sigma\left(\left(L^{\infty}\right)^{*}, \mathbb{L}^{\infty}\right)$-lower semi-continuity of $V$. Since $\langle\mu, \xi\rangle=\left\langle\mu_{1}, \xi\right\rangle$ if $\xi$ is $\mathcal{G}$-measurable, this shows that assertion (ii) of the proposition holds true for every sub- $\sigma$-algebra $\mathcal{G} \supset \sigma(\xi)$.

3. Let $Z_{1}$ be the density with respect to $\mathbb{P}$ of the regular part $\mu_{1}^{r}$ of the measure $\mu_{1}=\mathbb{E}[\mu \mid \mathcal{G}]$. To prove (i), let $\nu_{\xi}$ be a random variable defined on $(\Omega, \mathcal{F}, \mathbb{P})$, uniformly distributed on $(0,1)$, such that $-\xi=q_{-\xi}\left(\nu_{\xi}\right)$. Define $Z_{2}:=q_{Z_{1}}\left(\nu_{\xi}\right)$ and $\mu_{2}:=Z_{2} \cdot \mathbb{P}+\mu^{s}$. Observe that $Z_{1}={ }_{d} Z_{2}$ and $\mu_{2} \sim_{c}-\xi$. Then, whenever the pair $(-\mu, \xi)$ is not comonotone, it follows from the law-invariance of $U$ and $V$ that $U(\xi)=\langle\mu, \xi\rangle+V(\mu)>\left\langle\mu_{2}, \xi\right\rangle+V\left(\mu_{2}\right) \geq U(\xi)$. This contradiction shows that (i) holds true. The final assertion of the Lemma follows from (ii), and the fact that $-h(\xi):=-\mathbb{E}[\mu \mid \xi]$ is comonotone with $\xi$ iff the measurable function $h: \mathbb{R} \longrightarrow \mathbb{R}$ can be chosen to be non-decreasing.

We next specialize the discussion to the comonotone case. Recall from Theorem 2.3 that comonotone law-invariant monetary utility functions have a quantile representation in terms of a concave non-decreasing function in $\overline{\mathcal{C}} \backslash$. For $\mu \in\left(\mathbb{L}^{\infty}\right)^{*}$, we shall denote

$$
\varphi_{\mu}(0):=0 \quad \text { and } \quad \varphi_{\mu}(t):=\left\|\mu^{s}\right\|_{\mathrm{ba}}-\int_{0}^{t} q_{-Z}(\alpha) d \alpha, \quad 0 \leq t \leq 1,
$$


where $\mu=Z \cdot \mathbb{P}+\mu^{s}$ is the decomposition of $\mu$ into a regular part, with density $Z$ with respect to $\mathbb{P}$, and a singular part $\mu^{s}$.

Lemma 4.2. Let $U(\xi):=\int q_{\xi}(t) \bar{\varphi}^{\prime}(t) d t, \xi \in \mathbb{L}^{\infty}$, for some $\bar{\varphi} \in \overline{\mathcal{C}} \backslash$. Then

$$
\partial U(\xi)=\left\{\mu \in\left(\mathbb{L}^{\infty}\right)^{*}: \varphi_{\mu} \in \overline{\mathcal{C}} \searrow, \varphi_{\mu} \leq \bar{\varphi} \text { and } q_{\xi} \text { is flat on }\left\{\varphi_{\mu}<\bar{\varphi}\right\}\right\}
$$

Proof. Let $\mu_{0} \in \partial U(\xi)$. By definition, the super-gradient of $U$ consists of all minimizers in the variational representation of Theorem 2.3, i.e.

$$
U(\xi)=\inf _{\mu \in\left(\mathbb{L}^{\infty}\right)^{*}}\langle\mu, \xi\rangle+V(\mu)=\left\langle\mu_{0}, \xi\right\rangle+V\left(\mu_{0}\right) .
$$

By Lemma 4.1, we have $\mu_{0} \sim_{c}-\xi$. Let $\nu_{\xi}$ be a random variable defined on $(\Omega, \mathcal{F}, \mathbb{P})$ uniformly distributed on $(0,1)$ such that $\xi q_{\xi}\left(\nu_{\xi}\right)$, and set $Z_{1}:=q_{Z}\left(\nu_{\xi}\right), \mu_{1}:=Z_{1} \cdot \mathbb{P}+\mu_{0}^{s}$. Then arguing as in Lemma 4.1, it follows from (4.3) that $\int_{0}^{1} q_{\xi}(t) \varphi_{\mu_{0}}^{\prime}(t) d t=\int_{0}^{1} q_{\xi}(t) \bar{\varphi}^{\prime}(t) d t$, which provides by a direct integration by parts :

$$
\int_{0}^{1}\left(\varphi_{\mu_{0}}-\bar{\varphi}\right)(t) d q_{\xi}(t)+\left(q_{\xi}(0+)-q_{\xi}(0)\right)\left(\varphi_{\mu_{0}}-\bar{\varphi}\right)(0+)=0 .
$$

By Theorem 2.3, $\varphi_{\mu_{0}} \leq \bar{\varphi}$. Then, the above equality holds if and only if $q_{\xi}$ is flat on $\left\{\varphi_{\mu_{0}}<\bar{\varphi}\right\}$.

Remark 4.1. Comparing with Carlier and Dana [12] and Föllmer and Schied [25], we see that comonotone law-invariant monetary utility functions are identified with convex distortions of probability measures, or equivalently with concave homogeneous Choquet integrals. In particular, the above Lemma 4.2 is a slight extension of Corollary 2 in [12] (they only consider the case where $\bar{\varphi}$ is a strictly concave increasing differentiable function with $\bar{\varphi}(0)=0$ and $\bar{\varphi}(1)=1$ so that the singular part of the elements of the subgradient are zero). In the present context, it is however important to make sure that $\partial U(\xi) \neq \emptyset$, so that it is necessary to define this super-gradient on the topological dual $\left(\mathbb{L}^{\infty}\right)^{*}$ instead of $\mathbb{L}^{1}(\mathbb{P})$. When both monetary utility functions are comonotone and law-invariant, there is no guarantee for the existence of an element of the super-gradient in $\mathbb{L}^{1}(\mathbb{P})$, whenever the associated concave function $\bar{\varphi} \in \overline{\mathcal{C}} \backslash$ exhibits a jumps at zero.

We are now ready for the

Proof of Proposition 3.1 (i) $\Longrightarrow$ (ii) By Theorem 3.1, $\left(\xi_{0}, \xi_{1}\right) \in \mathbb{A}(X)$ is a Pareto optimal allocation if and only if $-\xi_{i} \in \partial V_{i}(\bar{\mu})$ for some $\bar{\mu} \in\left(\mathbb{L}^{\infty}\right)^{*}$.

1. We first observe that this implies that $-X \in \partial\left(V_{0}+V_{1}\right)(\bar{\mu})$, and by the Fenchel duality relation, $\bar{\mu} \in \partial\left(U_{0} \square U_{1}\right)(X)$. By Lemma 4.1, we deduce that $\mu:=\mathbb{E}[\bar{\mu} \mid X] \in \partial\left(U_{0} \square U_{1}\right)(X)$, which implies that $\mu \in \partial U_{0}\left(\xi_{0}\right) \cap \partial U_{1}\left(\xi_{1}\right)$ by Lemma 6.2 below. By Lemma 4.1 (ii), this implies that $-\mu \sim_{c} \xi_{0}$ and $-\mu \sim_{c} \xi_{1}$. 
2. Since $\mu \in \partial U_{i}\left(\xi_{i}\right)$, we have $U_{i}\left(\xi_{i}\right)=\int_{0}^{1} q_{\xi_{i}}(t) \varphi_{\mu}^{\prime}(t) d t$ for $i=0,1$. By Lemma 2.2, this shows that $\left(\xi_{0}, \xi_{1}\right)$ is a Pareto optimal allocation if and only if

$$
\int_{0}^{1} q_{X}(t)\left(\bar{\varphi}_{0} \wedge \bar{\varphi}_{1}\right)^{\prime}(t) d t=\int_{0}^{1}\left(q_{\xi_{0}}+q_{\xi_{1}}\right)(t) \varphi_{\mu}^{\prime}(t) d t=\int_{0}^{1} q_{X}(t) \varphi_{\mu}^{\prime}(t) d t
$$

where we used the fact that $q_{\xi_{0}}+q_{\xi_{1}}=q_{\xi_{0}+\xi_{1}}$ as $\xi_{0} \sim_{c} \xi_{1}$. Integrating by parts, this provides the equivalent characterization :

$$
\int_{0}^{1}\left(\varphi_{\mu}-\bar{\varphi}_{0} \wedge \bar{\varphi}_{1}\right) d q_{X}(t)=0 .
$$

Since $\varphi_{\mu} \leq \bar{\varphi}_{0} \wedge \bar{\varphi}_{1}$ by Lemma 4.2 , this implies that $\varphi_{\mu}=\bar{\varphi}_{0} \wedge \bar{\varphi}_{1}$ on $B:=\left\{d q_{X}()>0.\right\}$, and

$$
q_{\xi_{i}} \text { is flat on } B \cap\left\{\varphi_{\mu}<\bar{\varphi}_{i}\right\}=B \cap\left\{\bar{\varphi}_{1-i}<\bar{\varphi}_{i}\right\} \text { for each } i=0,1 .
$$

For $t \in B^{c} \cap\left\{\bar{\varphi}_{1}<\bar{\varphi}_{0}\right\}$, it follows from Lemma 4.2 that $\varphi_{\mu}(t)<\bar{\varphi}_{0}(t)$ and $q_{\xi_{0}}$ is flat at the point $t$. Recalling that $q_{\xi_{0}}+q_{\xi_{1}}=q_{X}$ by the comonotonicity of $\left(\xi_{0}, \xi_{1}\right)$, and the definition of $B^{c}$, this implies that $q_{\xi_{1}}$ is also flat at the point $t$. Applying the same argument for points in $B^{c} \cap\left\{\bar{\varphi}_{0}<\bar{\varphi}_{1}\right\}$, we see that

$$
q_{\xi_{0}} \text { and } q_{\xi_{1}} \text { are flat on } B^{c} \cap\left\{\bar{\varphi}_{0} \neq \bar{\varphi}_{1}\right\} \text {. }
$$

Hence (4.4) is equivalent to (4.5)-(4.6).

(ii) $\Longrightarrow$ (i) Let $\nu_{X}$ be a random variable on $(\Omega, \mathcal{F}, \mathbb{P})$ uniformly distributed on $(0,1)$ such that $X=q_{X}\left(\nu_{X}\right)$. Let $\left(\xi_{0}, \xi_{1}\right)$ be a pair in $\mathbb{A} \nearrow(X)$ satisfying (ii). Then $\xi_{i}=f_{i}(X)$ for some non-decreasing maps $f_{i}, i=0,1$. Set $\bar{\varphi}:=\bar{\varphi}_{0} \wedge \bar{\varphi}_{1}$, and define $\mu:=\bar{\varphi}(0+) \delta_{0}\left(\nu_{X}\right)+$ $\bar{\varphi}^{\prime}(U) \mathbf{1}(0,1](U)$, where $\delta_{0}$ is the Dirac mass at 0 . By construction, we have $\varphi_{\mu}=\bar{\varphi}$ with the notation of (4.2), and we immediately check that $\varphi_{\mu} \leq \bar{\varphi}_{i}, i=0,1$, and $-\mu$ increases with $\xi_{0}$ and $\xi_{1}$. By Lemma 4.2 , it follows that $\mu \in \partial U_{0}\left(\xi_{0}\right) \cap \partial U_{1}\left(\xi_{1}\right)$ so that $\left(\xi_{0}, \xi_{1}\right)$ satisfies the first order condition for the optimization problem $U_{0} \square U_{1}$.

\section{$5 \quad$ Strict risk aversion conditionally on lower-tail events}

This section is dedicated to the proof of Proposition 3.2. The crucial arguments are isolated in the two following lemmas.

Lemma 5.1. Let $U$ be a law-invariant monetary utility function which is strictly risk averse conditionally on lower tail events. Let $(\xi, Z) \in \mathbb{L}^{\infty} \times \mathbb{L}^{1}(\mathbb{P})$ be such that $Z \in \partial U(\xi)$. Assume that $\mathbb{P}[Z=\operatorname{ess}-\sup Z]>0$. Then $\xi$ is a.s. constant on the $\operatorname{set}\{Z=\operatorname{ess}-\sup Z\}$.

Proof. Let $(\xi, Z) \in \mathbb{L}^{\infty} \times \mathbb{L}^{1}(\mathbb{P})$ with $Z \in \partial U(\xi)$. By Theorem 2.1 and Lemma 4.1, we have

$$
U(\xi)=\mathbb{E}[Z \xi]+V(Z) \text { and }-Z \sim_{c} \xi
$$


Suppose further that the set $A:=\{Z=$ ess-sup $Z\}$ has positive $P$-measure. In order to prove the required result, we shall assume to the contrary that $\xi$ is not constant on $A$, and work towards a contradiction. Since $(\xi,-Z)$ is comonotone, it follows that $A \in \mathcal{F}^{\downarrow}(\xi)$. Since $U$ is strictly risk averse conditionally on lower tail events, we conclude that

$$
U(\xi)<U(\tilde{\xi}) \quad \text { where } \quad \tilde{\xi}:=\xi \mathbf{1}_{A^{c}}+\mathbb{E}[\xi \mid A] \mathbf{1}_{A} .
$$

Observing that $\mathbb{E}[Z \tilde{\xi}]=\mathbb{E}[Z \xi]$, it follows from the concavity of $U$ that

$$
U(\tilde{\xi}) \leq U(\xi)+\mathbb{E}[Z(\tilde{\xi}-\xi)]=U(\xi),
$$

thus contradicting (5.2).

Lemma 5.2. Let $U$ be a strictly monotone monetary utility function, and consider some pair $(\xi, Z) \in \mathbb{L}^{\infty} \times \mathbb{L}^{1}(\mathbb{P})$ with $Z \in \partial U(\xi)$. Then $Z>0 \mathbb{P}-$ a.s.

Proof. For $(\xi, Z) \in \mathbb{L}^{\infty} \times \mathbb{L}^{1}(\mathbb{P})$ with $Z \in \partial U(\xi)$, and $A:=\{Z=0\}$, it follows from the concavity of $U$ that $U\left(\xi+\mathbf{1}_{A}\right) \leq U(\xi)+\mathbb{E}\left[Z \mathbf{1}_{A}\right]=U(\xi)$. This implies that $\mathbb{P}[A]=0$ by the strict monotonicity of $U$.

We are now ready for the

Proof of Proposition 3.2 Let $\left(\xi_{0}, \xi_{1}\right) \in \mathbb{A} \nearrow(X)$ be a Pareto optimal allocation. By Theorem 3.1, there exists some $\mu \in\left(\mathbb{L}^{\infty}\right)^{*}$ such that

$$
\mu \in \partial U_{0}\left(\xi_{0}\right) \cap \partial U_{1}\left(\xi_{1}\right) .
$$

1. We first prove that $\left(\xi_{0}, \xi_{1}\right)=\left(-(X-\kappa)^{-}, \kappa \vee X\right)$ as in the statement of the proposition, for some $\kappa \in \mathbb{R}$. By Lemma 4.1, we have $-\mu \sim_{c} \xi_{i}, i=0,1$, and the associated nondecreasing concave function satisfies

$$
\varphi_{\mu}(t) \leq \bar{\varphi}_{0}(t):=\left(\alpha^{-1} t\right) \wedge 1, \quad t \in[0,1] .
$$

In particular, this implies that $\varphi_{\mu}(0+)=0$, and therefore $\mu=Z \cdot \mathbb{P}$ for some $Z \in \mathbb{L}^{1}(\mathbb{P})$. Moreover, since $U_{1}$ is strictly monotone, it follows from the comonotonicity of the pairs $\left(-Z, \xi_{i}\right), i=0,1$, together with Lemma 5.2 that $Z>0 \mathbb{P}$-a.s. Then, there exists $\beta \in[0,1)$ such that $\left\{\varphi_{\mu}<\bar{\varphi}_{0}\right\}=(\beta, 1)$. By Lemma 4.2, we see that

$$
q_{\xi_{0}} \text { is constant on }[\beta, 1] \text {. }
$$

Since $U_{1}$ is strictly risk-averse conditionally on lower tail events, it follows again from the comonotonicity of the pairs $\left(-Z, \xi_{i}\right), i=0,1$, together with Lemma 5.1 that

$$
q_{\xi_{1}} \text { is constant on }[0, \beta] \text {. }
$$

Since $q_{X}=q_{\xi_{0}}+q_{\xi_{1}}$, by the comonotonicity of the pair $\left(\xi_{0}, \xi_{1}\right)$. By (5.4) and (5.5), the Pareto allocation $\left(\xi_{0}, \xi_{1}\right)=\left(-(X-\kappa)^{-}, \kappa \vee X\right)$ as in the statement of the proposition, 
for some $\kappa \in[$ ess-inf $X$, ess- $\sup X]$.

2. It remains to prove the uniqueness statement. By the previous step, all Pareto optimal allocations are of the form $\left(\xi_{0}(\kappa), \xi_{1}(\kappa)\right)=\left(-(X-\kappa)^{-}, \kappa \vee X\right)$ for some $\kappa \in$ [ess-inf $X$, ess- sup $X$ ]. Suppose that $\left(\xi_{0}(\kappa), \xi_{1}(\kappa)\right)$ and $\left(\xi_{0}\left(\kappa^{\prime}\right), \xi_{1}\left(\kappa^{\prime}\right)\right)$ are two Pareto optimal allocations for some $\kappa, \kappa^{\prime} \in[$ ess-inf $X$, ess- sup $X]$. Then, $2\left(\bar{\xi}_{0}, \bar{\xi}_{1}\right):=\left(\xi_{0}(\kappa), \xi_{1}(\kappa)\right)$ $+\left(\xi_{0}\left(\kappa^{\prime}\right), \xi_{1}\left(\kappa^{\prime}\right)\right)$ is also a Pareto optimal allocation, and therefore $\left(\bar{\xi}_{0}, \bar{\xi}_{1}\right)=\left(\xi_{0}\left(\kappa^{\prime \prime}\right), \xi_{1}\left(\kappa^{\prime \prime}\right)\right)$ for some $\kappa^{\prime \prime} \in$ [ess-inf $X$, ess-sup $X$ ] by the first part of this proof. This in turn implies that $\left(\xi_{0}(\kappa), \xi_{1}(\kappa)\right)=\left(\xi_{0}\left(\kappa^{\prime}\right), \xi_{1}\left(\kappa^{\prime}\right)\right)=\left(\xi_{0}\left(\kappa^{\prime \prime}\right), \xi_{1}\left(\kappa^{\prime \prime}\right)\right)$ a.s.

\section{Existence of Pareto optimal allocations for law-invariant monetary utility functions}

\subsection{A duality argument}

We first prove the existence of a Pareto optimal allocation in $\mathbb{A}(X)$ by means of duality arguments. This provides a partial proof of Theorem 3.2 which states in addition that a Pareto optimal allocation exists in the smaller set $\mathbb{A} \nearrow(X)$.

By Theorem 3.1, for given $X \in \mathbb{L}^{\infty}$, Pareto optimal allocations are characterized as the solutions of the maximization problem

$$
U_{0} \square U_{1}(X)=\sup _{\left(\zeta_{0}, \zeta_{1}\right) \in \mathbb{A}(X)} U_{0}\left(\zeta_{0}\right)+U_{1}\left(\zeta_{1}\right) .
$$

Recall from Section 2.2 that $\operatorname{dom}\left(U_{0} \square U_{1}\right)=\mathbb{L}^{\infty}$ for law-invariant monetary utility functions $U_{0}$ and $U_{1}$. Also, since $U_{0} \square U_{1}$ is a monetary utility function, it is continuous with respect to the $\mathbb{L}^{\infty}$-norm, and therefore $\left(\mathbb{L}^{\infty}\right)^{*} \supset \partial\left(U_{0} \square U_{1}\right)(X) \neq \emptyset$, see Moreau [34].

Since $U_{0} \square U_{1}$ and $V_{0}+V_{1}$ are conjugate, see Remark 2.2, we know from the Fenchel duality relation that $\mu \in \partial\left(U_{0} \square U_{1}\right)(X)$ iff $-X \in \partial\left(V_{0}+V_{1}\right)(\mu)$.

We next claim that :

$$
\partial\left(V_{0}+V_{1}\right)(\mu)=\partial V_{0}(\mu)+\partial V_{1}(\mu)
$$

A direct proof of this claim for the case of law-invariant monetary utility functions is possible but somewhat technical. For ease of presentation, we report in Subsection 6.2 a direct proof for the existence of Pareto optimal allocations, which avoids the passage to the $\left(\mathbb{L}^{1},\left(\mathbb{L}^{\infty}\right)^{*}\right)$ duality, and provides as a by-product the existence of a Pareto optimal allocation in the smaller set $\mathbb{A} \nearrow(X)$. As a by-product, we obtain Lemma 6.2 which states that the existence statement is actually equivalent to the identity (6.2) which, in particular, yields a proof for (6.2). Notice that that the law-invariance assumption cannot be dropped, as shown in Subsection 6.3 below. 
We then deduce from (6.2) the existence of a pair $\left(\xi_{0}, \xi_{1}\right) \in \mathbb{L}^{\infty} \times \mathbb{L}^{\infty}$ such that $\xi_{0}+\xi_{1}=$ $X$ and $-\xi_{i} \in \partial V_{i}(\mu)$ for $i=0,1$. Using again the Fenchel duality relation, we conclude that $\mu \in \partial U_{0}\left(\xi_{0}\right) \cap \partial U_{1}\left(\xi_{1}\right)$, which precisely says that the pair $\left(\xi_{0}, \xi_{1}\right)$ solves the first order condition for the problem (6.1), see Theorem 3.1. Hence, $\left(\xi_{0}, \xi_{1}\right)$ is a Pareto optimal allocation.

\subsection{A direct existence argument}

In this section, we provide a complete proof of Theorem 3.2, and we obtain as a by-product a justification of (6.2).

We first start by proving that the maximization problem in the definition of the supconvolution $U_{0} \square U_{1}$ can be restricted to pairs $\left(\xi_{0}, \xi_{1}\right) \in \mathbb{A} \nearrow(X)$.

Lemma 6.1. Let $U_{0}$ and $U_{1}$ be two law-invariant monetary utility functions. Then

$$
U_{0} \square U_{1}(X)=\sup _{\left(\xi_{0}, \xi_{1}\right) \in \mathbb{A} \nearrow(X)} U_{0}\left(\xi_{0}\right)+U_{1}\left(\xi_{1}\right)
$$

Proof. * We first recall that any attainable allocation $\left(\xi_{0}, \xi_{1}\right) \in \mathbb{A}(X)$ is dominated by some comonotone attainable allocation $\left(\hat{\xi}_{0}, \hat{\xi}_{1}\right) \in \mathbb{A}(X)$ in the sense of second order stochastic dominance. This result was proved by Landsberger and Meilijson [33] in the context of a finite probability space, and further extended to $\mathbb{L}^{\infty}$ allocations in a general probability space by Dana and Meilijson [14].

By Corollary 4.59 in [25], any law-invariant monetary utility function preserves second order stochastic dominance, which provides

$$
U_{i}\left(\hat{\xi}_{i}\right) \geq U_{i}\left(\xi_{i}\right)
$$

Hence the maximization problem in the definition of $U_{0} \square U_{1}$ can be restricted to subset of $\mathbb{A}(X)$ consisting of comonotone pairs $\left(\xi_{0}, \xi_{1}\right)$. As in Section 3.2 above, we conclude that this subset is precisely $\mathbb{A} \nearrow(X)$.

We are now ready for the

Proof of Theorem 3.2 Let $\left(\xi_{0}^{n}, \xi_{1}^{n}\right)_{n}$ be a maximizing sequence of $U_{0} \square U_{1}(X)$. By Lemma 6.1, we can find such a maximizing sequence in $\mathbb{A}^{\nearrow}(X)$ with $\xi_{0}^{n}=f_{0}^{n}(X)$ and $\xi_{1}^{n}=f_{1}^{n}(X)$ for some non-decreasing functions $f_{0}^{n}, f_{1}^{n}:[a, b] \longrightarrow \mathbb{R}$, where $[a, b]:=$ [ess-inf $X$, ess-sup $X$ ] is the range of the (bounded) random variable $X$. By possibly adding a constant to $\xi_{0}^{n}$, we may assume ess-inf $\xi_{0}^{n}=0$. From the equality $\xi_{0}^{n}+\xi_{1}^{n}=X$, we see that $0 \leq f_{0}^{n} \leq b-a$ and $a \leq f_{1}^{n} \leq b$. Hence, for each $n \geq 1, f_{0}^{n} \in \mathcal{B}:=\{f:[a, b] \longrightarrow \mathbb{R}$,

\footnotetext{
${ }^{*}$ We are grateful to Beatrice Acciaio for pointing out to us a gap in a previous proof of this lemma.
} 
$|f| \leq b-a, f$ and Id $-f$ are non-decreasing $\}$. Observe that any function in $\mathcal{B}$ is 1 -Lipschitz continuous. Then $\mathcal{B}$ is a bounded closed equicontinuous family, and it follows from the Ascoli Theorem that, after possibly passing to a subsequence, $f_{0}^{n} \longrightarrow f_{0}$ uniformly on $[a, b]$ for some $f_{0} \in \mathcal{B}$. This implies that $f_{1}^{n} \longrightarrow f_{1}:=\mathrm{Id}-f_{0}$ uniformly on $[a, b]$. By the $\mathbb{L}^{\infty}$-continuity of $U_{0}$ and $U_{1}$, we have $U_{0} \square U_{1}(X)=\lim _{n \rightarrow \infty} U_{0}\left(\xi_{0}^{n}\right)+U_{1}\left(\xi_{1}^{n}\right)=$ $U_{0}\left(f_{0}(X)\right)+U_{1}\left(f_{1}(X)\right)$ so that $\left(f_{0}(X), f_{1}(X)\right)$ is the required maximizer.

We conclude this section by proving that the crucial identity (6.2) is in fact equivalent to the existence of Pareto optimal allocation, which has been proved in Subsection 6.2 under the law-invariance assumption. With this result, this justifies the duality-based existence argument reported in the beginning of Section 6 .

Lemma 6.2. Let $U_{0}, U_{1}$ be two concave functions from $\mathbb{L}^{\infty}$ to $\mathbb{R}$, and assume that $U_{0} \square U_{1}$ is upper semi-continuous and $\operatorname{dom}\left(U_{0} \square U_{1}\right)=\mathbb{L}^{\infty}$. Then, the following statements are equivalent :

(i) for all $X \in \mathbb{L}^{\infty}, U_{0} \square U_{1}(X)=U_{0}\left(\xi_{0}\right)+U_{1}\left(\xi_{1}\right)$ for some $\left(\xi_{0}, \xi_{1}\right) \in \mathbb{A}(X)$,

(ii) for all $\mu \in\left(\mathbb{L}^{\infty}\right)^{*}$, we have $\partial\left(V_{0}+V_{1}\right)(\mu)=\partial V_{0}(\mu)+\partial V_{1}(\mu)$.

Proof. (ii) $\Longrightarrow($ i) : Under (ii), the argument in the beginning of this section provides the required existence result.

(i) $\Longrightarrow\left(\right.$ ii) : For $\mu \in\left(\mathbb{L}^{\infty}\right)^{*}$, the inclusion $\partial V_{0}(\mu)+\partial V_{1}(\mu) \subseteq \partial\left(V_{0}+V_{1}\right)(\mu)$ is always true. If $\partial\left(V_{0}+V_{1}\right)(\mu)=\emptyset$, then the previous inclusion implies that $\partial V_{0}(\mu)+\partial V_{1}(\mu)=$ $\partial\left(V_{0}+V_{1}\right)(\mu)=\emptyset$. We next assume that $\partial\left(V_{0}+V_{1}\right)(\mu) \neq \emptyset$, and we intend to prove that, under (i), $\partial\left(V_{0}+V_{1}\right)(\mu) \subseteq \partial V_{0}(\mu)+\partial V_{1}(\mu)$. For $X \in \partial\left(V_{0}+V_{1}\right)(\mu)$, it follows from (i) that $U_{0} \square U_{1}(X)=U_{0}\left(\xi_{0}\right)+U_{1}\left(\xi_{1}\right)$ for some $\left(\xi_{0}, \xi_{1}\right) \in \mathbb{A}(X)$. From the proof of (ii) $\Longleftrightarrow$ (iii) of Theorem 3.1 together with Remark 3.2, we see that $V_{i}(\mu)=U_{i}\left(\xi_{i}\right)-\left\langle\mu, \xi_{i}\right\rangle, i=0,1$. Hence $\xi_{i} \in \partial V_{i}(\mu)$ and therefore $X=\xi_{0}+\xi_{1} \in \partial V_{0}(\mu)+\partial V_{1}(\mu)$.

\subsection{Law-invariance and existence of Pareto optimal allocations}

We now show that the assumption of law-invariance in Theorem 3.2 can not be dropped.

Example 6.1. This example was triggered by a question of N. El Karoui, and shows that, even in a finite-dimensional setting, it may happen that the set of Pareto optimal allocations is empty for any possible aggregate risk $X \in \mathbb{L}^{\infty}$. Let $U_{1}$ be given by the entropic utility function : $U_{1}(\xi):=-\ln \mathbb{E}[\exp (-\xi)], \xi \in \mathbb{L}^{\infty}$. It is well-known that the domain of its conjugate is included in $\mathbb{L}^{1}(\mathbb{P})$, and $V_{1}(Z):=\mathbb{E}[Z \ln Z] \chi_{\mathcal{Z}}(Z), Z \in \mathbb{L}^{1}$.

Let $Z_{0}$ be any density in $\mathcal{Z}$ with $\mathbb{P}\left[Z_{0}=0\right]>0$, and such that $V_{1}\left(Z_{0}\right)<\infty$. One checks that $\partial V_{1}\left(Z_{0}\right)=\emptyset$. Define the utility of Agent 0 by $U_{0}(\xi):=\mathbb{E}\left[Z_{0} \xi\right], \xi \in \mathbb{L}^{\infty}$. The domain of its conjugate is included in $\mathbb{L}^{1}(\mathbb{P})$, and $V_{0}(Z):=\chi_{\left\{Z_{0}\right\}}(Z), Z \in \mathbb{L}^{1}$. 
Since $Z_{0} \in \operatorname{dom}\left(V_{0}\right) \cap \operatorname{dom}\left(V_{1}\right)$, it follows from Lemma 2.1 that $\operatorname{dom}\left(U_{0} \square U_{1}\right)=\mathbb{L}^{\infty}$. Given a total risk $X \in \mathbb{L}^{\infty}$, it follows from 3.1 (iii) that any Pareto optimal allocation $\left(\xi_{0}, \xi_{1}\right) \in \mathbb{A}(X)$ must satisfy $\xi_{i} \in \partial V_{i}(Z)$, which implies that $Z=Z_{0}$ and $\xi_{1} \in \partial V_{1}\left(Z_{0}\right)$. This can not happen as $\partial V_{1}\left(Z_{0}\right)=\emptyset$.

Example 6.2. In this example due to F. Delbaen [20], we show that the set of Pareto optimal allocations might be empty in the context where all monetary utility functions $U_{0}, U_{1}, U_{0} \square U_{1}$, are positively-homogeneous, and have the Fatou property.

Let $\left\{\left(B_{t}^{0}, B_{t}^{1}\right), t \geq 0\right\}$ be a standard Brownian motion in $\mathbb{R}^{2}$ defined on $(\Omega, \mathcal{F}, \mathbb{F}, \mathbb{P})$, where $\mathbb{F}=\left\{\mathcal{F}_{t}, t \geq 0\right\}$ is the natural filtration (with the usual conditions) generated by $\left(B^{0}, B^{1}\right)$. For $i=0,1$, let $\mathcal{M}^{i}$ denote the subset of $\mathbb{L}^{1}(\mathbb{P})$ formed by the densities of the probability measures $Q$, absolutely continuous with respect to $\mathbb{P}$, such that $B^{i}$ is a Brownian motion under $Q$. The monetary utility functions $U_{i}$ are defined by

$$
U_{i}(\xi):=\inf _{Q \in \mathcal{M}^{i}} \mathbb{E}^{Q}[\xi], \quad \xi \in \mathbb{L}^{\infty}(\mathbb{P}), \quad i=0,1
$$

It is shown in $[20]$ that

$$
U_{i}(\xi)=\max \left\{a: a+\left(H \cdot B^{i}\right)_{\infty} \leq \xi \text { for some } H \in \mathbb{H}^{i}\right\} \quad \text { and } \quad U_{0} \square U_{1}(\xi)=\mathbb{E}^{\mathbb{P}}[\xi],
$$

where $\mathbb{H}^{i}$ is the collection of all predictable $B^{i}$-integrable processes such that the process $\left\{\left(H \cdot B^{i}\right)_{t}, t \geq 0\right\}$ is bounded.

Define the total risk

$$
X:=B_{T}^{0}+B_{T}^{1} \quad \text { where } \quad T:=\inf \left\{t:\left|B_{t}^{0}+B_{t}^{1}\right|=1\right\} .
$$

Clearly $X \in \mathbb{L}^{\infty}$ and $U_{0} \square U_{1}(X)=\mathbb{E}^{\mathbb{P}}[X]=0$. We now suppose that one can find a pair $\left(\xi_{0}, \xi_{1}\right) \in \mathbb{A}(X)$ such that $U_{0}\left(\xi_{0}\right)+U_{1}\left(\xi_{1}\right)=0$, and work towards a contradiction.

We may assume without loss of generality that $U_{0}\left(\xi_{0}\right)=U_{1}\left(\xi_{1}\right)=0$ so that

$$
\left(H^{0} \cdot B^{0}\right)_{\infty}+\left(H^{1} \cdot B^{1}\right)_{\infty} \leq X \quad \text { for some }\left(H^{0}, H^{1}\right) \in \mathbb{H}^{0} \times \mathbb{H}^{1} .
$$

On the other hand, we know from the martingale representation theorem that the only $B$-integrable predictable integrands $K=\left(K^{0}, K^{1}\right)$ such that $K^{i} \cdot B^{i}$ are bounded in $\mathbb{L}^{2}(\mathbb{P})$ and $K \cdot B \leq X$ are (up to indistinguishability) $K^{0}=K^{1}=\mathbf{1}_{(0, T]}$. Hence we must have $H^{0}=H^{1}=\mathbf{1}_{(0, T]}$, which is in contradiction with (6.4) as $H^{i} \notin \mathbb{H}^{i}$.

\section{APPENDIX : Proof of Lemma 3.1}

(i) For $\xi \in \mathbb{L}^{\infty}$, and $A \in \mathcal{F}^{\downarrow}(\xi)$, set $\tilde{\xi}:=\xi \mathbf{1}_{A^{c}}+\mathbb{E}[\xi \mid A] \mathbf{1}_{A}$, and observe that there exists $\alpha>0$ such that the $\mathbb{P}$-distribution of $(\xi, \tilde{\xi})$ is the $\lambda$-distribution of $\left(q_{\xi}, q_{\tilde{\xi}}\right)$, where

$$
q_{\tilde{\xi}}:=q_{\xi} \mathbf{1}_{[\alpha, 1]}+Q_{\xi}(\alpha) \mathbf{1}_{[0, \alpha)} \text { and } Q_{\xi}(\alpha):=\frac{1}{\alpha} \int_{0}^{\alpha} q_{\xi}(s) d s .
$$


We then directly compute that

$$
U(\tilde{\xi})-U(\xi)=Q_{\xi}(\alpha) \int_{0}^{\alpha} \bar{\varphi}^{\prime}(s) d s-\int_{0}^{\alpha} q_{\xi}(s) \bar{\varphi}^{\prime}(s) d s .
$$

This shows that, whenever $\bar{\varphi}^{\prime}$ is constant near the origin, there exists $\xi \in \mathbb{L}^{\infty}$ such that $U(\tilde{\xi})-U(\xi)=0$ for sufficiently small $\alpha$. Conversely, assume that $\bar{\varphi}$ is not linear on $[0, \alpha]$ for every $\alpha \in(0,1]$, and let us show that $U$ is strictly risk averse conditionally on lower tail events. Set

$$
\psi(t):=\left(\int_{0}^{\alpha} \bar{\varphi}^{\prime}(s) d s\right)^{-1} \int_{0}^{t} \bar{\varphi}^{\prime}(s) d s \text { for } t \in[0, \alpha],
$$

and observe that $\psi(0)=0, \psi(\alpha)=1, \psi$ is continuous, concave on $[0, \alpha]$, and $\psi$ is not linear. Then $\psi(t) \geq \alpha^{-1} t, 0 \leq t \leq \alpha$, and $\lambda\left(\left\{t \in[0, \alpha]: \psi(t)>\alpha^{-1} t\right\}\right)>0$. Since $q_{\xi}(0)<q_{\xi}(\alpha)$, It follows from an integration by parts that

$$
\begin{aligned}
U(\tilde{\xi})-U(\xi) & =\int_{0}^{\alpha} \bar{\varphi}^{\prime}(s) d s\left(Q_{\xi}(\alpha)+\int_{0}^{\alpha} \bar{\psi}(s) d q_{\xi}(s)+\psi(0+)\left(q_{\xi}(0+)-q_{\xi}(0)\right)-\left[q_{\xi} \psi\right]_{0}^{\alpha}\right) \\
& \geq \int_{0}^{\alpha} \bar{\varphi}^{\prime}(s) d s\left(Q_{\xi}(\alpha)+\int_{0}^{\alpha} \bar{\psi}(s) d q_{\xi}(s)-\left[q_{\xi} \psi\right]_{0}^{\alpha}\right) \\
& >\int_{0}^{\alpha} \bar{\varphi}^{\prime}(s) d s\left(Q_{\xi}(\alpha)+\int_{0}^{\alpha} \alpha^{-1} s d q_{\xi}(s)-\left[q_{\xi}(s) \alpha^{-1} s\right]_{0}^{\alpha}\right)=0 .
\end{aligned}
$$

(ii) For $\xi, \zeta \in \mathbb{L}^{\infty}$, it follows from the super-linearity of $U$ that $U(\xi+\zeta) \geq U(\xi)+U(\zeta)$. Then $U$ is strictly monotone if and only if $U(\xi)>0$ for every $\xi \in \mathbb{L}_{+}^{\infty} \backslash\{0\}$. For any such a $\xi$, let $\beta:=\inf \left\{s>0: q_{\xi}(s)>0\right\}$. Since $U(\xi)=\int_{\beta}^{1} q_{\xi}(s) \bar{\varphi}^{\prime}(s) d s$, the required equivalence follows from the fact that $\bar{\varphi}^{\prime}(1)=1$ and $\bar{\varphi}$ is continuous at the point 1 .

\section{References}

[1] Arrow K.J. (1963). Uncertainty and the welfare of medical care. American Economic Review 53, 941-973.

[2] Artzner P., Delbaen F., Eber J.M. and D. Heath (1999). Coherent measures of risk. Mathematical Finance 9, 203-228.

[3] Barrieu P. and N. ElKaroui (2002). Reinsuring climatic risk using optimally designed weather bonds. Geneva Papers, Risk and Insurance Theory 27, 87-113.

[4] Barrieu P. and N. ElKaroui (2002). Optimal design of derivatives in illiquid markets. Quantitative Finance 2, 1-8.

[5] Barrieu P. and N. ElKaroui (2003). Optimal derivatives design and diversification in financial markets with non-tradable risk, preprint. 
[6] Barrieu P. and N. ElKaroui (2005). Inf-convolution of risk measures and optimal risk transfer. Finance and Stochastics 9, 269-298.

[7] Bühlmann, H. (1984). The general economic principle premium. Astin Bulletin 14, $13-21$.

[8] Bühlmann, H. and S. Jewell (1979). Optimal risk exchanges. Astin Bulletin 10, 243-262.

[9] Borch K. (1962). Equilibrium in a reinsurance market. Econometrica 30, 424-444.

[10] Brézis H. (1983). Analyse Fonctionelle, Masson.

[11] Camerer C. (2003). Behavioral Game Theory, Princeton University Press.

[12] Carlier G. and R.-A. Dana (2003). Core of convex distortions of probability. Journal of Economic Theory 113, 199-222.

[13] Dana R.-A. and M. Jeanblanc (2003). Financial Markets in Continuous Time, Springer.

[14] Dana R.-A. and I. Meilijson (2003). Modelling agents' preferences in complete markets by second order stochastic dominance. Peprint, Université Paris Dauphine.

[15] Dana R.-A. and M. Scarsini (2005). Optimal risk sharing with background risk. Preprint.

[16] Denneberg D. (1994). Non-additive measures and integrals. Kluwer Academic Publishers, Holland.

[17] Dhaene J., M. Denuit, M.J. Goovaerts, R. Kaas and D. Vyncke (2002). The concept of comonotonicity in actuarial science and finance: theory. Insurance: Mathematics and Economics 31, 3-33.

[18] Delbaen F. (2000). Coherent risk measures. Lecture Notes of Scuola Normale, Pisa.

[19] Delbaen F. (2002). Coherent measures of risk on general probability spaces. In Advances in Finance and Stochastics, Essays in Honor of Dieter Sondermann (K. Sandmann and P.J. Schonbucher, eds.) 1-37, Springer, Berlin.

[20] Delbaen F. (2005). Hedging bounded claims with bounded outcomes. Preprint.

[21] Frittelli M. and E.R. Rossaza-Gianin. Putting order in risk measures. Journal of Banking and Finance 26(7) (2002), 1473-1486.

[22] Frittelli M. and E.R. Rossaza-Gianin (2005). Law invariant convex risk measures. Advances in Mathematical Economics 7, 33-46. 
[23] Fischer T. (2001). Examples of coherent risk measures depending on one-sided moments. Preprint.

[24] Föllmer H. and A. Schied (2002). Convex measures of risk and trading constraints. Finance and Stochastics 6, 429-448.

[25] Föllmer H. and A. Schied (2004).Stochastic Finance, Second Edition, de Gruyter.

[26] Gerber H.U. (1979). An Introduction to Mathematical Risk Theory. Huebner Foundation Monograph 8, Wharton School, University of Pennsylvania.

[27] Heath D. and H. Ku (2004). Pareto equilibria with coherent measures of risk, Mathematical Finance 14(2), 163-172.

[28] Jouini E. and C. Napp (2004). Conditional comonotonicity, avec Clotilde Napp. To appear in Decisions in Economics and Finance.

[29] Jouini E., Schachermayer W. and N. Touzi (2005). Law-invariant risk measures have the Fatou property. To appear in Advances in Mathematical Economics.

[30] Joseph H., Boyd R., Bowles S. Camerer C., Gintis H., McElreath R., and Fehr E. (2001). In search of Homo economicus : Experiments on 15 Small-Scale Societies, American Economic Review 91, 73-79.

[31] Klöppel S. and M. Schweizer (2005). Dynamic Utility Indifference Valuation via Convex Risk Measures. Preprint.

[32] Kusuoka S. (2001). On law-invariant coherent risk measures. Advances in Mathematical Economics 3, 83-95.

[33] Landsberger, M. and I Meilijson (1994). Comonotone allocations, Bickel-Lehmann dispersion and the Arrow-Pratt measure of risk aversion. Annals of Operations Research, 52, 97-106.

[34] Moreau J.J. Fonctionnelles convexes (1966), Séminaire Equations aux dérivées partielles. Collg̀e de France.

[35] Nowak M.A., Page K., and Sigmund K. (2000). Fairness versus reason in the ultimatum game, Science 289, 1773-1775.

[36] Rockafellar R.T. (1997). Convex Analysis, Princeton Landmarks in Mathematics, Princeton University Press. 